\title{
Kinematical and static force analysis on redundant drive wire mechanism with velocity constraint modules to reduce the number of actuators
}

\author{
Tam Nhat Le ${ }^{*+}$, Hiroki Dobashi ${ }^{\dagger}$ and Kiyoshi Nagai ${ }^{\dagger}$
}

\begin{abstract}
This study proposes new wire mechanism called "the redundant drive wire mechanism" (RDWM), driven by double actuator modules. The RDWM is configured with velocity constraint modules (VCMs) that provide high-acceleration global and fine local motions. Suitable RDWM candidates are found by combining the kinematic analysis with static force analysis. The study also clarifies the role of VCMs in reducing the required number of actuators while keeping the orientation of the top plate. We propose a judgment procedure with three steps: a static force analysis in the whole motion space, a kinematic analysis that finds the active constraint space wherein the top plate can produce velocity and a static force analysis in this active constraint space. The proposed judgment procedure and the role of VCMs are validated by examples.
\end{abstract}

Keywords: Wire mechanism, Redundant drive, Double actuator module, Velocity constraint module, RDWM

\section{Background}

The structure of wire mechanisms are often employed to configure fast mechanisms. Wire mechanisms reduce the inertia of the top plate, enabling large acceleration motions. For example, the wire driven method called "FALCON" was configured in a high-speed manipulator [1], with a peak acceleration of $43 \mathrm{G}$. In this method, the redundant drive concept was applied in a parallel mechanism to produce a large resultant force. The "NINJA" mechanism [2] was rendered light-weight by arranging the motors on a base. Its top plate with six degrees of freedom (DOF) was driven by four sub-arms with a parallel link structure. The design parameters of the mechanism were optimized while reducing the inertia of the top plate. In experiments, the encoders computed that "NINJA" can accelerate to $\geq 100 \mathrm{G}$. The mechanisms of "DELTA" [3] and "HEXA" [4] were based on similar concepts. Later, Nagai et al. [5] introduced a high-speed

\footnotetext{
*Correspondence: letamritsumei@gmail.com

${ }^{\dagger}$ All authors contributed equally to this work

Department of Robotics, College of Science and Engineering,

Ritsumeikan University, 1-1-1 Noji-higashi, 525-8577 Kusatsu, Shiga, Japan
}

parallel mechanism for electronic part mounters, known as "the constrained differential drive mechanism" (CDDM). They analyzed a typical four-DOF pick-andplace motion and divided the trajectory into two regions, $\mathrm{A}$ and $\mathrm{B}$. In region $\mathrm{A}$, high-precision motions were produced, whereas high-acceleration motions were produced in region $B$. Thereafter, they integrated CDDM and the virtual force redundancy (VFR) concept into a highspeed parallel mechanism. Experimentally, this mechanism accelerate to $\geq 20 \mathrm{G}$. Recently, a mechanism known as "the capturing robot" was introduced, which achieved accelerations of $100 \mathrm{G}$ [6] by utilizing the spring energy in the pre-shaping dynamics of the link fingers. However, high-acceleration motions were limited to one direction when moving to grasp an object.

The configurations of mechanisms with separated parts for global and local motions have also been studied. Osumi and colleagues [7] and [8] installed a manipulator on the top plate. In this mechanism, the top plate produced the global motion, whereas the changing pose of the manipulator produced the local motion. Lampariello et al. [9] introduced their robot named "KUKA," which 
produces similar motions by two manipulators installed on a platform. However, installing the actuators or manipulators on the top plate impacts a very high inertia to the top plate, reducing the capability for high-acceleration global motions.

To judge whether wire tension can constrain the top plate and whether the conventional wire mechanism can generate an omnidirectional resultant force, Cong Pham et al. $[10,11]$ adopted the force closure condition [12] in the workspace analysis. They proposed an algorithm that determines the optimal workspace with the optimal tension factor value, thereby simplifying the analysis.

Following an investigation of these topics, we began seeking a mechanism that yields high-acceleration motion, a mechanism that generates both global and local motions, and a method for judging the configurations of wire mechanisms. The redundant drive wire mechanism (RDWM) with double actuator modules (DAMs) for realizing fast and fine motion has been already proposed [13]. The DAM is a pair of actuators that control the length or tension of a single wire, generating a large translational force that greatly accelerates the top plate. Because the actuators are positioned outside of the top plate, the top plate develops low inertia and achieves high-acceleration motions. In addition, fine-local motions are generated on the top plate. However, RDWM requires a large number of actuators: at least eight in the planar case and 14 in the 3D case.

To reduce the number of actuators, we previously introduced a velocity constraint module (VCM) into RDWM [14]. The VCM applies the same velocities to different points of the top plate, then constrains the orientation around a certain axis. This constraint is irrelevant to the RDWM because the orientation of the end effector can be adjusted by a local mechanism. The literature has confirmed the strong performance of VCM in the planar case, but the 3D case requires further investigation. Moreover, methodologies for judging the RDWM candidates remain insufficiently studied.

Therefore, this study investigates the reduction in the number of actuators by introducing VCMs to RDWM in the 3D case, not merely in the planar case. It then discusses the judgment procedure, which assesses whether RDWM candidate configurations generate the desired motion space. The remainder of the paper is organized as follows. First, the basic structure and basic concept of RDWM with and without VCMs is introduced and the technical problems of introducing VCMs into RDWM are discussed. Second, the procedure for judging candidate RDWMs with and without VCMs is proposed. Third, the judgment procedure is validated in numerical examples of planar and 3D cases. These examples also confirm the role of the VCMs in reducing the number of actuators while keeping the orientation of the top plate. The study findings are summarized in the "Conclusion" section.

\section{Problem statement \\ Basic structure of RDWM}

In the RDWM concept, the DAMs are used to achieve high-acceleration and high precision-motions, and a 1DOF RDWM has been already proposed [13]. The DAM contains two actuators that move the top plate and rotate the local pulley, as shown in Fig. 1. When the two actuators rotate in the same direction, a global translational motion is produced. Conversely, when the two actuators rotate in different directions, local pulley rotates to generate precise local motion. As shown in Fig. 2, the top plate in RDWM in RDWM is controlled by fourteen wires in seven DAMs. The RDWM can move at high speed through a large working space and also undertake specific tasks with high precise using the three fingers on its top plate. The fingers perform grasping tasks using a common DOF and can hold the object using two DOFs for each finger. The structure of a planar RDWM using four DAMs is shown in Fig. 3. Global, high-acceleration motions of the top plate are achieved by applying a large translational force to the mechanism. This force is the sum of the two wire tensions of each DAM. The difference between the wire tensions of each DAM generates a torque for the corresponding local pulley, enabling precise local motions of the top plate mechanism. The basic equations of the planar RDWM using four DAMs are given below:

$$
\begin{aligned}
& \boldsymbol{F}=\boldsymbol{W T}, \\
& \dot{\boldsymbol{i}}=W^{\mathrm{T}} \boldsymbol{v},
\end{aligned}
$$

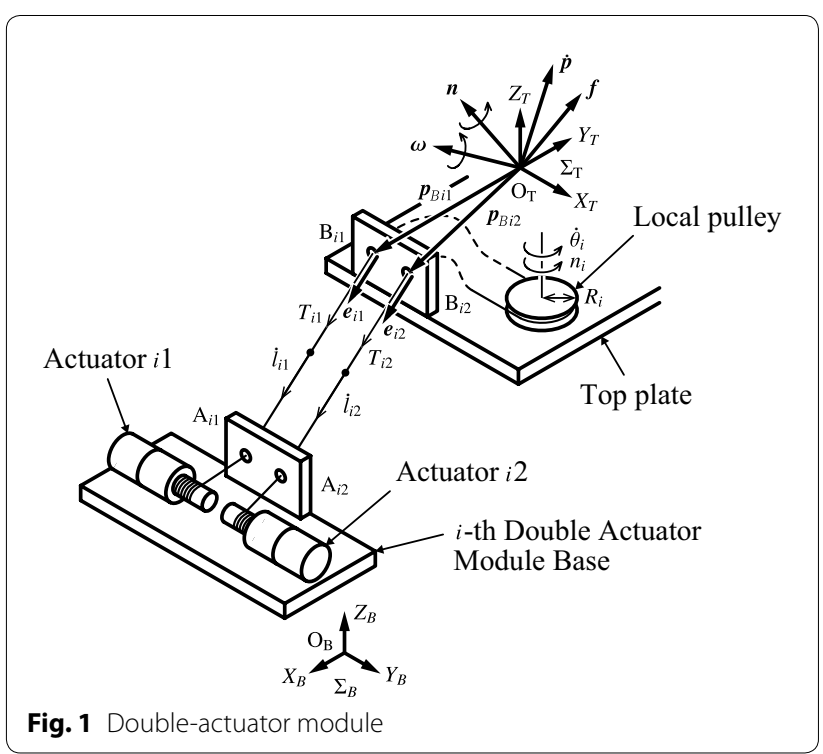




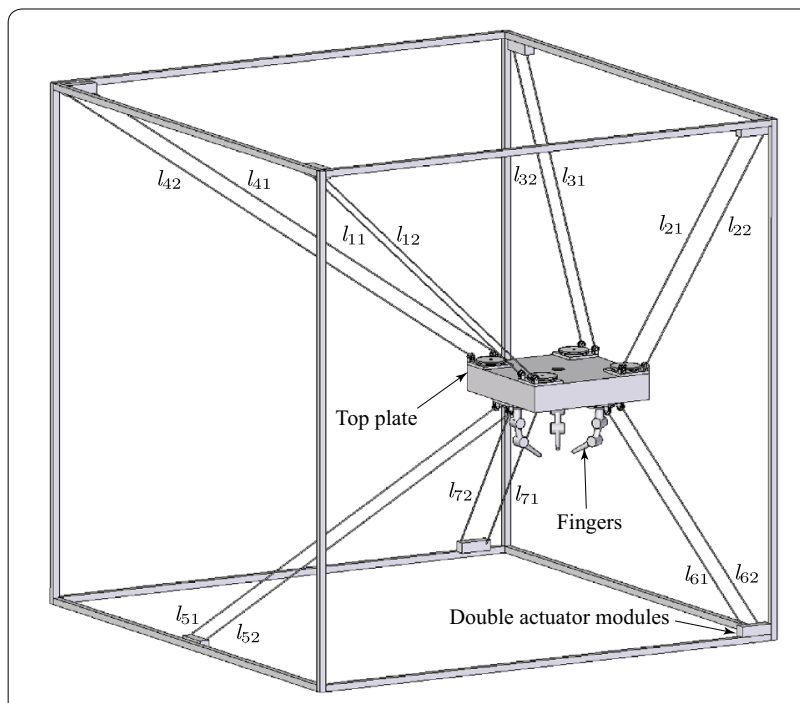

Fig. 2 Image of the target wire mechanism

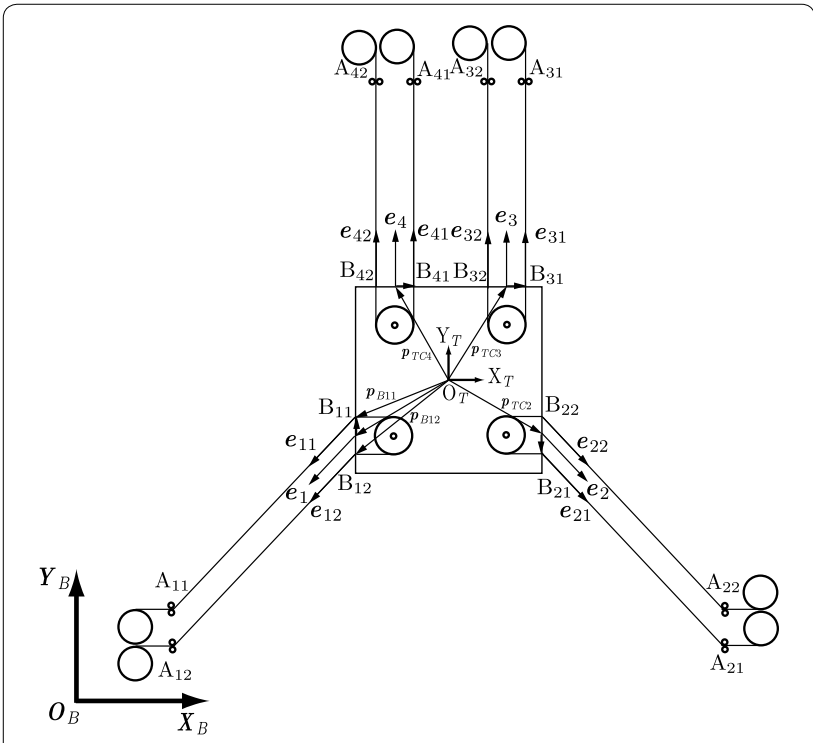

Fig. 3 Planar RDWM with four DAMs

where: $\quad \boldsymbol{F}=\left[f_{x} f_{y} n_{z} n_{1} n_{2} n_{3} n_{4}\right]^{\mathrm{T}} \in \boldsymbol{R}^{7}$ is the resultant force vector. Here $f_{x}$ and $f_{y}$ are the resultant forces in the $\mathrm{X}$ and $\mathrm{Y}$ directions, respectively, for global motion of the top plate; $n_{z}$ is the resultant moment around the Z-axis for global motion of the top plate; and $n_{1}, n_{2}, n_{3}, n_{4}$ are torques on the four local pulleys.

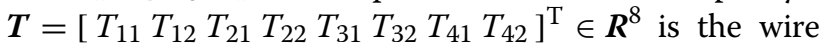
tension vector, where $T_{i j}(i=1,2,3,4 ; j=1,2)$ is the tension on each wire, and $\dot{\boldsymbol{l}}=\left[\dot{l}_{11} \dot{l}_{12} \dot{l}_{21} \dot{l}_{22} \dot{l}_{31} \dot{l}_{32} \dot{l}_{41} \dot{l}_{42}\right]^{\mathrm{T}} \in \boldsymbol{R}^{8}$ is the wire velocity vector, where $\dot{l}_{i j}(i=1,2,3,4 ; j=1,2)$ is the velocity of each wire. $\boldsymbol{v}=\left[\dot{p}_{x} \dot{p}_{y} \dot{\phi}_{z} \dot{\theta}_{1} \dot{\theta}_{2} \dot{\theta}_{3} \dot{\theta}_{4}\right]^{\mathrm{T}} \in \boldsymbol{R}^{7}$ is the output velocity vector, where $\dot{p}_{x}$ and $\dot{p}_{y}$ are the top plate's velocities in the $\mathrm{X}$ and $\mathrm{Y}$ directions respectively, $\dot{\phi}_{z}$ is the top plate's angular velocity around the Z-axis, $\dot{\theta}_{1}, \dot{\theta}_{2}, \dot{\theta}_{3}$ and $\dot{\theta}_{4}$ are the local pulleys' angular velocities, and $\boldsymbol{W} \in \boldsymbol{R}^{7 \times 8}$ is the wire matrix, which can be derived from Eq. (75).

The basic equations of actuators are represented as follows:

$$
\begin{aligned}
& \dot{\boldsymbol{i}}=\boldsymbol{J} \dot{\boldsymbol{q}}, \\
& \boldsymbol{\tau}=\boldsymbol{J}^{\mathrm{T}} \boldsymbol{T},
\end{aligned}
$$

where $\dot{\boldsymbol{q}}=\left[\dot{q}_{11} \dot{q}_{12} \dot{q}_{21} \dot{q}_{22} \dot{q}_{31} \dot{q}_{32} \dot{q}_{41} \dot{q}_{42}\right]^{\mathrm{T}} \in \boldsymbol{R}^{8}$ is the actuator velocity vector. Here $\dot{q}_{i j}(i=1,2,3,4 ; j=1,2)$ is angular velocity of each actuator, and $\boldsymbol{\tau}=\left[\tau_{11} \tau_{12}\right.$ $\left.\tau_{21} \tau_{22} \tau_{31} \tau_{32} \tau_{41} \tau_{42}\right]^{\mathrm{T}} \in \boldsymbol{R}^{8}$ is the actuator torque vector, where $\tau_{i j}(i=1,2,3,4 ; j=1,2)$ is the torque of each actuator. $\boldsymbol{J}=$ diag. $\left(N_{11}, N_{12}, \ldots, N_{42}\right) \in \boldsymbol{R}^{8 \times 8}$ is a regular matrix of reduction ratios $N_{i j}(i=1,2,3,4 ; j=1,2)$ of actuator $i j$ th which include the pulley radius information. $\boldsymbol{J}$ is actually the Jacobian matrix between the wire and actuator velocities.

\section{Basic concept of RDWM with VCM}

Figure 4a shows a VCM with a single actuator module. The VCM is characterized by equality of the two wire velocities $\dot{l}_{i 1}$ and $\dot{l}_{i 2}$, which restricts the posture of the top plate around the axis perpendicular to the plane containing the two wires. The VCM requires one fewer actuator than driving the top plate in the same direction by two single wires.

Similarly, the VCM is combined with DAM to generate high acceleration motions is shown in Fig. 4b. Here, the two summation velocities $\dot{l}_{i}$ and $\dot{l}_{i+1}$ are equal because $\dot{l}_{i 1}=\dot{l}_{(i+1) 1}$ and $\dot{l}_{i 2}=\dot{l}_{(i+1) 2}$. This configuration restricts the posture of the top plate around the axis perpendicular to the plane containing the four wires. This VCM requires two fewer actuators than driving the top plate in the same direction by two DAMs.

The above analysis shows that configuring VCMs on RDWM can reduce the required number of actuators. Moreover, the VCMs constraint the posture of the top plate. Therefore, we can propose candidate RDWMs with VCMs that fix the posture of the top plate and allow translational-only motions with fewer actuators than other configurations. However, this research excludes the vibration of the mechanism and investigations on vibration suppression. The wire is assumed as ideally stiffness with no wire elasticity.

Figure 5 shows the basic concept of RDWM with VCM. As shown in this figure, the top plate can move at fast speed through a large working space, but its orientation is fixed by the VCMs, which have parallel alignments of two sets of double wires. However, the orientation of the top plate is intrinsically difficult to change, and the end point 


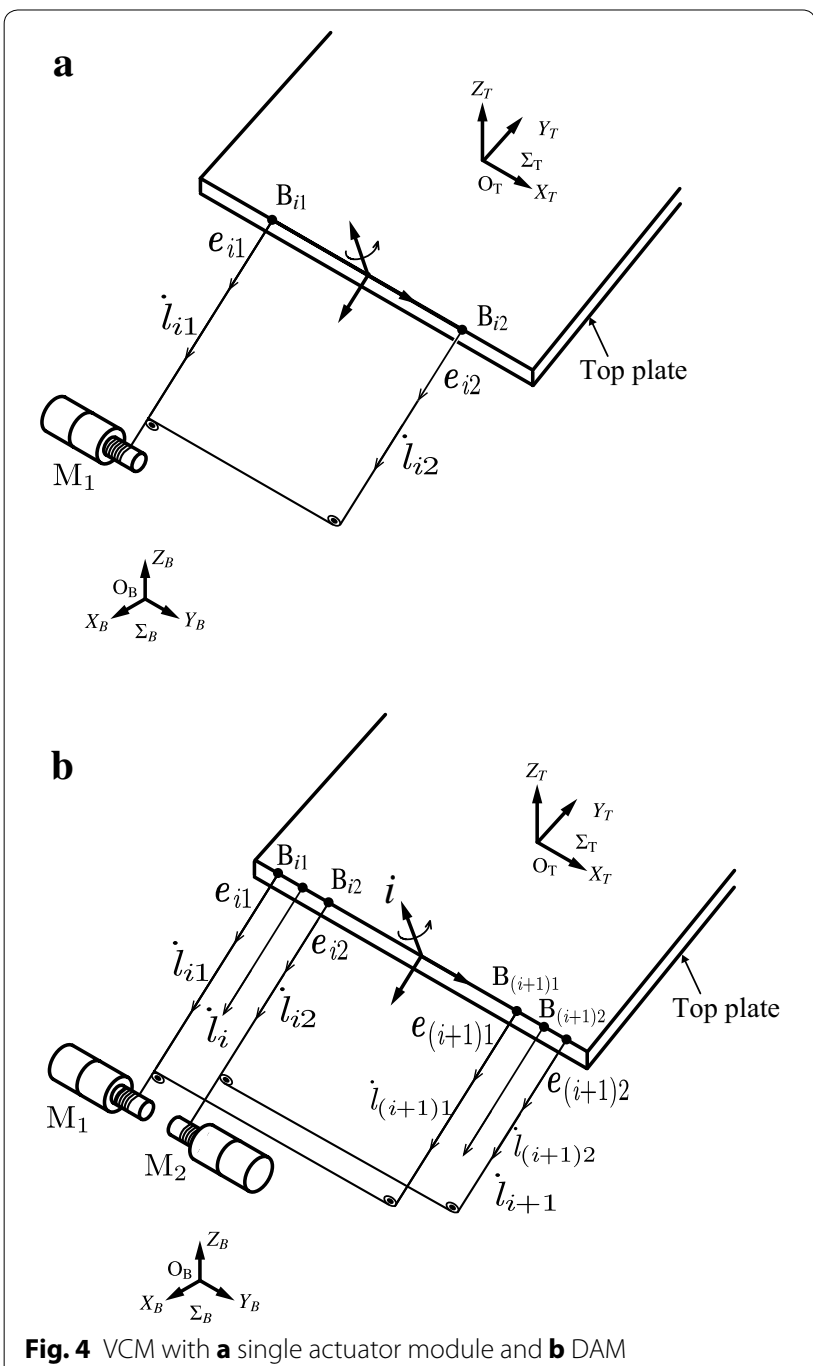

of the RDWM can be reoriented by fine motions generated by the local mechanism in the proposed structure. Figure 7 shows the proposed structure of the planar RDWM with a VCM. The structure is governed by Eqs. (1) and (2) and the following basic equations of the actuators:

$$
\begin{aligned}
& \dot{\boldsymbol{l}}=\boldsymbol{J}_{v c} \dot{\boldsymbol{q}}_{v c}, \\
& \boldsymbol{\tau}_{v c}=\boldsymbol{J}_{v c}^{\mathbf{T}} \boldsymbol{T},
\end{aligned}
$$

where $\quad \dot{\boldsymbol{q}}_{v c}=\left[\dot{q}_{11} \dot{q}_{12} \dot{q}_{21} \dot{q}_{22} \dot{q}_{31} \dot{q}_{32}\right]^{\mathrm{T}} \in \boldsymbol{R}^{6} \quad$ is the actuator velocity vector. Here $\dot{q}_{i j}(i=1,2,3 ; j=1,2)$ is the angular velocity of each actuator. $\boldsymbol{\tau}_{v c}=\left[\begin{array}{ll}\tau_{11} & \tau_{12}\end{array}\right.$ $\left.\tau_{21} \tau_{22} \tau_{31} \tau_{32}\right]^{\mathrm{T}} \in \boldsymbol{R}^{6}$ is the actuator torque vector, where $\tau_{i j}(i=1,2,3 ; j=1,2)$ is the torque on each actuator, and $J_{v c} \in \boldsymbol{R}^{8 \times 6}$ is the Jacobian matrix between the wire and actuator velocities.

In the numerical test cases (see later), the VCMs reduce the number of required actuator units from 8 to 6 in the planar RDWM and from 14 to 8 in the 3D RDWM.

\section{Technical problems to be solved}

There is an important difference between Eqs. (3), (4) and (5), (6). The latter pair of equations expresses the constraint imposed by the VCMs. Unlike $\boldsymbol{J}$, the matrix $\boldsymbol{J}_{v c}$ is not regular because it contains the constraint; therefore, the wire tensions are interdependent. The kinematics equations of the whole mechanism are derived from the set of Eqs. (2), (3) and the set of Eqs. (2), (5) for the planar RDWM with DAMs and planar RDWM with VCM, respectively:

$$
\begin{aligned}
& J \dot{\boldsymbol{q}}=\boldsymbol{W}^{\mathrm{T}} \boldsymbol{v}, \\
& \boldsymbol{J}_{v c} \dot{\boldsymbol{q}}_{v c}=\boldsymbol{W}^{\mathrm{T}} \boldsymbol{v},
\end{aligned}
$$

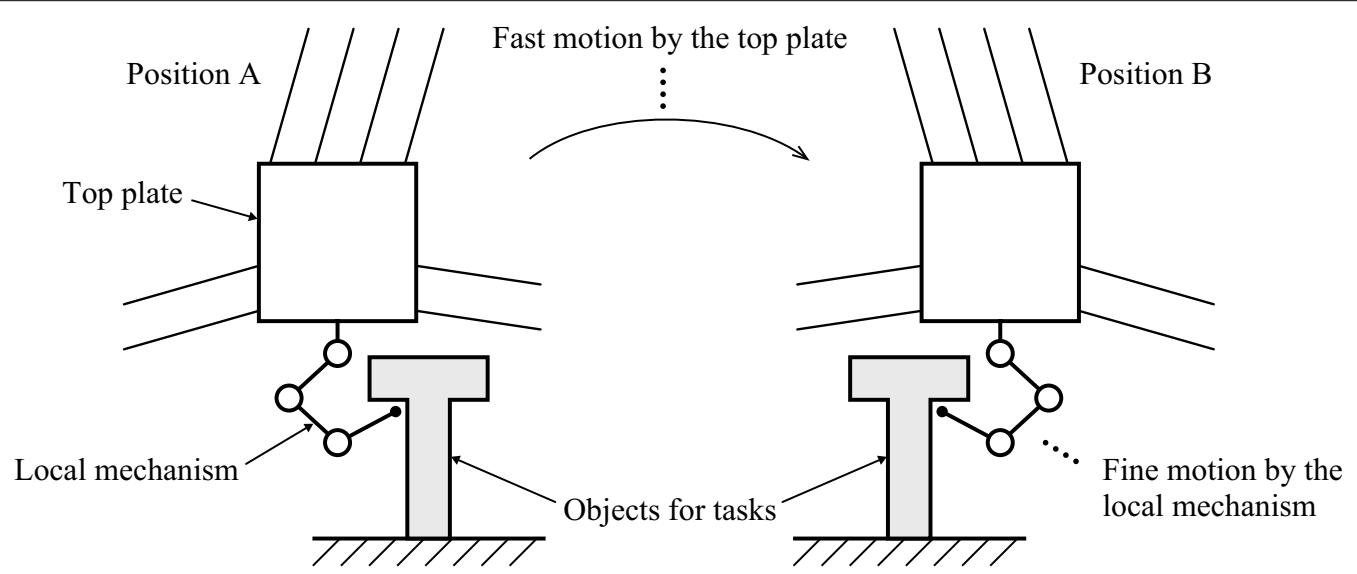

Fig. 5 Basic concept of RDWM with VCM 
from Eq. (7), it is easily to derive $\dot{\boldsymbol{q}}=\boldsymbol{J}^{-1} \boldsymbol{W}^{\mathrm{T}} \boldsymbol{v}$ and the velocity of actuator unit $\dot{\boldsymbol{q}}$ can be got because the matrix $\boldsymbol{J} \in \boldsymbol{R}^{8 \times 8}$ is a regular one and is invertible. However, considering Eq. (8): it cannot be directly solved as Eq. (7) because the matrices $\boldsymbol{J}_{v c} \in \boldsymbol{R}^{8 \times 6}$ is not regular and is not invertible. Therefore, the configuration of RDWM with VCM needs to use a kinematical analysis based on convex analysis to solve the problem.

The RDWM candidates cannot be judged solely by checking the vector closure condition (static force analysis). The space (directions and dimensions) of producible velocity of the top plate must be found by kinematic analysis because the VCM excludes the velocity from certain directions. In some cases, the space of producible velocity of the top plate can be intuited from geometrical considerations (see Fig. 7) as mentioned in the subsection "Basic concept of RDWM with VCM". However, in more general cases, this space must be found by an analytical method without relying on intuition. Whether the resultant global-motion force is generated in the desired direction within the space of producible velocity must then be judged by static force analysis. In stepwise fashion, these two analyses will find proper candidate RDWMs with/ without VCM. The kinematical analysis was conducted in our previous work [14] based on [16], but more considered combined analysis is presented here.

\section{Proposed judgment procedure for finding RDWM candidates with/without VCM}

\section{Outline of the procedure}

To solve the technical problems discussed in the previous section, we propose the following judgment procedure for finding RDWM candidates with/without VCM:

Step 1: Check the necessary condition for vector closure; Step 2: Find the space of producible velocity (also called the active constraint space $\boldsymbol{S}_{A C}$ ) by kinematical analysis; Step 3: Check the vector closure condition within the space of producible velocity.

The above judgment procedure is applicable to RDWM candidates with and without VCM. The contents of each step are described as below:

Using the vector closure condition, step 1 judges whether a resultant force in any direction on the top plate covers the whole motion space. This is a necessary condition.

By kinematical analysis, step 2 derives the active and passive constraint spaces, in which the top plate can and cannot acquire a velocity, respectively.

Using the vector closure condition, step 3 judges whether a resultant force in any direction on the top plate covers the active constraint space derived in step 2 .
The proposed judgment procedure can be demonstrated in simple planar configurations, as illustrated in Figs. 3, 7 and 8.

Intuitively, the top plate in Fig. 3 can move in the XOY plane and also rotate around the Z-axis. In step 1, the resultant force should be produced in the $\mathrm{X}$ and $\mathrm{Y}$ directions and around the Z-axis. Step 2, the velocity of the top plate should also be produced in the $\mathrm{X}$ and $\mathrm{Y}$ directions and around the Z-axis. In step 3, the resultant force force in the static force analysis should again be producible in the $\mathrm{X}$ and $\mathrm{Y}$ directions and around the $\mathrm{Z}$-axis.

Intuitively the top plate in Fig. 7 can move in the XOY plane but the VCM forbids its rotation around the $\mathrm{Z}$-axis. In step 1 , the resultant force should be produced in the $\mathrm{X}$ and $\mathrm{Y}$ directions and around the $\mathrm{Z}$-axis. The step 2 should restrict the velocity of the top plate to the $\mathrm{X}$ and $\mathrm{Y}$ directions. In step 3, the static force analysis should restrict the resultant force to the active constraint space (i.e., the $\mathrm{X}$ and $\mathrm{Y}$ directions).

Intuitively, the configuration of Fig. 8 cannot realize a positive resultant moment around the Z-axis, so appears improper. Step 1 fails to obtain a producible resultant force in all directions. In step 2, the velocity of the top plate should be producible in the $\mathrm{X}$ and $\mathrm{Y}$ directions and around the Z-axis. However, similar to the step 1, step 3 cannot find a resultant force that is produced in any direction.

\section{Details of step 1: checking the necessary condition for holding the vector closure}

Step 1 uses the vector closure condition $[1,17]$ (which is a necessary condition) to judge whether the resultant force can be produced in any direction through out the motion space of the mechanism. The contents of this condition are detailed below:

An RDWM with $n$ DOFs global motion of the top plate satisfies the vector closure condition if the wire matrix contributing to the resultant force on the top plate $W_{G}$ satisfies the following two conditions:

$$
\begin{aligned}
& \text { C1) } \operatorname{rank}\left(\boldsymbol{W}_{G}\right)=n \text {. } \\
& \text { C2) There exists a vector } \boldsymbol{T}>\mathbf{0} \text { that satisfies } \boldsymbol{W}_{G} \boldsymbol{T}=\mathbf{0} \text {. }
\end{aligned}
$$

For checking the vector closure condition, it is sufficient to derive the matrix $W_{G}$ by Eq. (23) because this matrix alone contributes to the resultant force for producing global motion of the top plate. The $\operatorname{rank}\left(W_{G}\right)$ in condition $\mathrm{C} 1$ defines the number of permitted directions of the resultant forces. If $\operatorname{rank}\left(W_{G}\right)$ is full $(=n)$ the resultant forces can be produced in the whole motion space of the mechanism. In contrast, a partial $\operatorname{rank}\left(W_{G}\right)$ denotes that the resultant force cannot be produced in one or more directions, so the mechanism configuration should be eliminated. 
Under condition $\mathrm{C} 2$, the matrix $W_{G}$ may not be regular because the sizes of wire tension vector $\boldsymbol{T}$ and the resultant force vector on the whole motion space $\boldsymbol{F}$ may differ. In this case, the number of roots exceeds the number of equations. If the candidate RDWM is feasible, a positive wire tension $\boldsymbol{T}$ such that $\boldsymbol{W}_{G} \boldsymbol{T}=\mathbf{0}$ will exist, meaning that the resultant force can be produced in any direction throughout the whole motion space. Any wire mechanism in which matrix $W_{G}$ satisfies both $\mathrm{C} 1$ and $\mathrm{C} 2$ will satisfy the vector closure condition, and the resultant force can be produced in any direction within the whole motion space.

\section{Details of step 2: find the producible velocity space by kinematical analysis (KA)}

The kinematical analysis judges the motion direction space of the RDWM candidates. This procedure first derives the velocities of the DAMs and VCMs contributing to the top plate's velocity. The derived velocity sets are then combined into a complete set of the top plate's velocities, which is converted from face-form to span-form by convex theory. The conversion procedure is summarized in Appendix 4 , and is comprehensively solved by linear programming in [18]. The kinematical analysis is detailed below:

\section{a. Set of top plate velocities contributed by the DAM without VCM}

Assuming that the wire velocities satisfy $-1[\mathrm{~m} / \mathrm{s}] \leq \dot{l}_{i j} \leq$ $1[\mathrm{~m} / \mathrm{s}]$, the DAM characteristic yield the following inequalities:

$$
\left\{\begin{array}{l}
-1 \leq \dot{l}_{i 1} \leq 1 \\
-1 \leq \dot{l}_{i 2} \leq 1
\end{array}\right.
$$

Eq. (9) implies that each wire can produce a non-zero velocity (either positive or negative). The values "1" and " -1 " are dummy values with no specific meaning. In matrix form, these inequalities are expressed as:

$$
\boldsymbol{A}_{L d i} \dot{\boldsymbol{l}}_{d i} \leq \boldsymbol{b}_{L d i}
$$

where

$$
\boldsymbol{A}_{L d i}=\left[\begin{array}{cc}
-1 & 0 \\
1 & 0 \\
0 & -1 \\
0 & 1
\end{array}\right] \in \boldsymbol{R}^{4 \times 2}, \quad \boldsymbol{b}_{L d i}=\left[\begin{array}{l}
1 \\
1 \\
1 \\
1
\end{array}\right] \in \boldsymbol{R}^{4} .
$$

The upper $2 \times 2$ block of $\boldsymbol{A}_{L d i}$ and the upper two rows of $\boldsymbol{b}_{L d i}$ in Eq. (11) correspond to the upper inequality related to $\dot{l}_{i 1}$ in Eq. (9). Similarly, the lower $2 \times 2$ block of $\boldsymbol{A}_{L d i}$ and the lower two rows of $\boldsymbol{b}_{L d d i}$ in Eq. (11) correspond to the lower inequality related to $\dot{l}_{i 2}$ in Eq. (9).

Considering the kinematic equation $\dot{\boldsymbol{l}}_{d i}=\boldsymbol{W}_{G i}^{\mathrm{T}} \boldsymbol{v}_{d i}$, the set of top plate velocities contributed by the DAM without $\mathrm{VCM}$ is given by

$$
\boldsymbol{V}_{d i}=\left\{\boldsymbol{v}_{d i} \mid \boldsymbol{A}_{V d i} \boldsymbol{v}_{d i} \leq \boldsymbol{b}_{V d i}\right\},
$$

where the matrix $\boldsymbol{A}_{V d i}$ in Eq. (12) is computed as

$$
\boldsymbol{A}_{V d i}=\boldsymbol{A}_{L d i} \boldsymbol{W}_{G i}^{\mathrm{T}},
$$

and the matrix $W_{G i}$ related to the global motion of the top plate of the DAM without VCM is given by

$W_{G i}=\left[\begin{array}{cc}{ }^{B} \boldsymbol{e}_{i} & { }^{B} \boldsymbol{e}_{i} \\ { }^{B} \boldsymbol{p}_{B i 1} \times{ }^{B} \boldsymbol{e}_{i} & { }^{B} \boldsymbol{p}_{B i 2} \times{ }^{B} \boldsymbol{e}_{i}\end{array}\right] \in \boldsymbol{R}^{n \times 2} ; \quad i=1, \ldots N_{D}$,

with ${ }^{B} \boldsymbol{e}_{i}={ }^{B} \boldsymbol{e}_{i j}(j=1,2)$. Mathematically, the symbol " $\times$ " in this equation is the operator to calculate the cross product of two vectors in $\boldsymbol{R}^{3}$ where each vector contains three elements of $\mathrm{X}, \mathrm{Y}$ and $\mathrm{Z}$ axes. However, in this paper, the symbol " $X$ " is also used to calculate the cross product of two vectors in $\boldsymbol{R}^{2}$ and the result of this product is a scalar. The detail of its derivation is shown in Appendix 1. Here, $n$ is the number of global motion DOFs of the top plate; $n=2$ in the 1D mechanism, $n=3$ in the planar mechanism and $n=6$ in the 3D mechanism.

\section{b. Set of top plate velocities contributed by the DAM with VCM}

Assuming that the wire velocities satisfy $-1[\mathrm{~m} / \mathrm{s}] \leq \dot{l}_{i j} \leq$ $1[\mathrm{~m} / \mathrm{s}]$ and considering the characteristic of $\mathrm{VCM}$, we obtain the following inequalities:

$$
\left\{\begin{array}{l}
-1 \leq \dot{l}_{i 1} \leq 1, \\
\dot{l}_{i 1}=\dot{l}_{(i+1) 1}, \\
-1 \leq \dot{l}_{i 2} \leq 1, \\
\dot{l}_{i 2}=\dot{l}_{(i+1) 2} .
\end{array}\right.
$$

Equation (14) implies that each wire can produce a nonzero velocity (either positive or negative), so the values " 1 " and " -1 " have no specific meaning. In matrix form, these inequalities are expressed as

$$
\boldsymbol{A}_{L v m} \dot{\boldsymbol{l}}_{v m} \leq \boldsymbol{b}_{L v m}
$$

where:

$$
\begin{aligned}
\boldsymbol{A}_{L v m}= & {\left[\begin{array}{cccc}
-1 & 0 & 0 & 0 \\
1 & 0 & 0 & 0 \\
1 & 0 & -1 & 0 \\
-1 & 0 & 1 & 0 \\
0 & -1 & 0 & 0 \\
0 & 1 & 0 & 0 \\
0 & 1 & 0 & -1 \\
0 & -1 & 0 & 1
\end{array}\right] \in \boldsymbol{R}^{8 \times 4} } \\
\boldsymbol{b}_{L v m} & =\left[\begin{array}{l}
1 \\
1 \\
0 \\
0 \\
1 \\
1 \\
0 \\
0
\end{array}\right] \in \boldsymbol{R}^{8} .
\end{aligned}
$$


In Eq. (16), the upper $2 \times 4$ block of $\boldsymbol{A}_{L v m}$ and the first two row of $\boldsymbol{b}_{L v m}$ correspond to the first inequality related to $\dot{l}_{i 1}$ in Eq. (14), and the second $2 \times 4$ block of $\boldsymbol{A}_{L v m}$ and the third and fourth rows of $\boldsymbol{b}_{L v m}$ correspond to the first equality related to $\dot{l}_{i 1}, \dot{l}_{(i+1) 1}$ in Eq. (14). Similarly, the third $2 \times 4$ block of $A_{L v m}$ and the fifth and sixth rows of $\boldsymbol{b}_{L v m}$ in Eq. (16) correspond to the second inequality related to $\dot{l}_{i 2}$ in Eq. (14), and the last $2 \times 4$ block of $\boldsymbol{A}_{L v m}$ and the final two rows of $\boldsymbol{b}_{L v m}$ correspond to the second equality related to Eq. (14).

Considering the kinematic equation, $\dot{\boldsymbol{l}}_{v m}=\boldsymbol{W}_{G V m}^{\mathrm{T}} \boldsymbol{v}_{v m}$, the set of top plate velocities contributed by the DAM with $\mathrm{VCM}$ is given by

$$
\boldsymbol{V}_{v m}=\left\{\boldsymbol{v}_{v m} \mid \boldsymbol{A}_{V v m} \boldsymbol{v}_{v m} \leq \boldsymbol{b}_{V v m}\right\},
$$

where the matrix $\boldsymbol{A}_{V v m}$ in Eq. (17) is computed as

$$
\boldsymbol{A}_{V v m}=\boldsymbol{A}_{L v m} \boldsymbol{W}_{G V m}^{\mathrm{T}}
$$

and the matrix $W_{G V m}$ related to the global motion of the top plate of the DAM with VCM is given by

$$
\begin{aligned}
\boldsymbol{W}_{G V m}= & {\left[\begin{array}{cccc}
{ }^{B} \boldsymbol{e}_{k} & { }^{B} \boldsymbol{e}_{k} & { }^{B} \boldsymbol{e}_{k} & { }^{B} \boldsymbol{e}_{k} \\
{ }^{B} \boldsymbol{p}_{B k 1} \times{ }^{B} \boldsymbol{e}_{k} & { }^{B} \boldsymbol{p}_{B k 2} \times{ }^{B} \boldsymbol{e}_{k}{ }^{B} \boldsymbol{p}_{B(k+1) 1} \times{ }^{B} \boldsymbol{e}_{k} & { }^{B} \boldsymbol{p}_{B(k+1) 2} \times{ }^{B} \boldsymbol{e}_{k}
\end{array}\right] } \\
& \in \boldsymbol{R}^{n \times 4} ; k=N_{D}+2 m-1 ; m=1, \ldots N_{V} .
\end{aligned}
$$

with ${ }^{B} \boldsymbol{e}_{k}={ }^{B} \boldsymbol{e}_{k j}=\boldsymbol{e}_{(k+1) j}(j=1,2)$. Here, $k$ is the ordering number of DAM without VCM when considering a DAM with VCM contains two DAMs without VCM.

\section{c. Face form of the producible velocity space}

Combining all velocity sets of the top plate contributed by all modules, the face form of the producible velocity space of the top plate is given by

$$
\boldsymbol{V}=\left\{\boldsymbol{v} \mid \boldsymbol{A}_{V} \boldsymbol{v} \leq \boldsymbol{b}_{V}\right\}
$$

where the velocity matrices of velocity of the top plate are defined as

$$
\begin{aligned}
& \boldsymbol{A}_{V}=\boldsymbol{A}_{L} \boldsymbol{W}_{G}^{\mathrm{T}}, \\
& \boldsymbol{b}_{V}=\left[\boldsymbol{b}_{L d 1}^{\mathrm{T}} \ldots \boldsymbol{b}_{L d N_{D}}^{\mathrm{T}} \boldsymbol{b}_{L v 1}^{\mathrm{T}} \ldots \boldsymbol{b}_{L v N_{V}}^{\mathrm{T}}\right]^{\mathrm{T}},
\end{aligned}
$$

where, the subscripts $L v$ and $L d$ denote the components belong to DAM with and without VCM; the subscripts $N_{V}$ and $N_{D}$ are the number of DAM with and without $\mathrm{VCM}$, respectively.

The matrix $\mathbf{A}_{L}$ is defined as

$$
\boldsymbol{A}_{L}=\operatorname{bdiag} .\left(\boldsymbol{A}_{L d 1}, \ldots, \boldsymbol{A}_{L d N_{D}}, \boldsymbol{A}_{L v 1}, \ldots, \boldsymbol{A}_{L v N_{V}}\right) \text {, }
$$

where bdiag. $\left(\boldsymbol{X}_{1}, \ldots, \boldsymbol{X}_{2}, \ldots, \boldsymbol{X}_{P}\right)$ denotes a blockdiagonal matrix constructed from the submatrices $\boldsymbol{X}_{1}, \boldsymbol{X}_{2}, \ldots, \boldsymbol{X}_{P}$ on its diagonal.
The contribution of matrix $W_{G}$ to the resultant force on the top plate is given by:

$\boldsymbol{W}_{G}=\left[\begin{array}{llllll}\boldsymbol{W}_{G 1} & \ldots & \boldsymbol{W}_{G N_{D}} & \boldsymbol{W}_{G V 1} & \ldots & \boldsymbol{W}_{G V N_{V}}\end{array}\right]^{\mathrm{T}} \in \boldsymbol{R}^{n \times 2\left(N_{D}+2 N_{V}\right)}$

where $W_{G i}$ and $W_{G V m}$ are obtained from Eqs. (13) and (18).

\section{d. Span form of the producible velocity space}

The face form of the producible velocity space does not clarify the directions and number of dimensions of the generated velocities. For this purpose, the face form Eq. (19) is converted to the following span form:

$$
\boldsymbol{V}=\left\{\sum_{t=1}^{\beta} \lambda_{t} \boldsymbol{v}_{t} \mid \sum_{t=1}^{\beta} \lambda_{t} \leq 1, \lambda_{t} \geq 0, t \in[1, \beta]\right\},
$$

using the method proposed in [18]. The results for all vertices are represented in the following matrix $A$ :

$$
A=\left[v_{1} \ldots v_{\beta}\right],
$$

where $\beta$ is the number of vertices.

Yoshikawa [19] mentioned the concepts of active and passive closure which are critically important for analyzing grasping and manipulation by robotic hands and the constraining mechanisms such as fixtures and vises. Passive and active closure refer to the ability of fixing and manipulating objects, respectively. In our research, the active constraint space $S_{A C}$ is defined as the space covered by the top plate velocities and movements. Conversely, the passive constraint space $S_{P C}$ is the space wherein the top plate cannot move. Mathematically, these spaces are defined as follows:

$$
S_{A C}=\mathcal{R}(A), S_{P C}=S_{A C}^{\perp}=\mathcal{N}\left(A^{\mathrm{T}}\right) .
$$

Details of step 3: check the vector closure condition within the producible velocity space by static force analysis (SFA)

The above mentioned kinematical analysis step obtains the active and passive constraint spaces $\left(\boldsymbol{S}_{A C}\right.$ and $\boldsymbol{S}_{P C}$, respectively). However, although the vector closure condition is a necessary condition in step 1 , this judgment is made over the whole motion space. Consequently, whether the resultant force can be produced in any direction within the active constraint space $S_{A C}$ is not confirmed. Therefore the SFA is used as the sufficient condition for judging RDWM candidates. The SFA procedure is detailed below.

First, the whole motion space coordinate is converted to the producible velocity space coordinate.

The static force relation between $\boldsymbol{F}_{G}$ and $\boldsymbol{T}$ is given by:

$$
\boldsymbol{F}_{G}=\boldsymbol{W}_{G} \boldsymbol{T},
$$


In the $2 \mathrm{D}$ case, $\boldsymbol{F}_{G}=\left[f_{x} f_{y} n_{z}\right]^{\mathrm{T}} \in \boldsymbol{R}^{3}$ is the resultant force vector that produces the global motion,

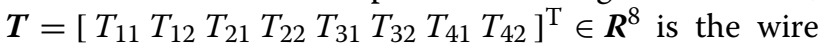
tension vector and the matrix $W_{G}$ is given by Eq. (23):

$$
\boldsymbol{W}_{G}=\left[\boldsymbol{W}_{G 1} \boldsymbol{W}_{G 2} \boldsymbol{W}_{G V 1}\right]^{\mathrm{T}} \in \boldsymbol{R}^{3 \times 8} .
$$

The matrices $\boldsymbol{W}_{G i}$ and $\boldsymbol{W}_{G V 1}$ are derived from Eqs. (13) and (18), respectively:

$\boldsymbol{W}_{G i}=\left[\begin{array}{cc}{ }^{B} \boldsymbol{e}_{i} & { }^{B} \boldsymbol{e}_{i} \\ { }^{B} \boldsymbol{p}_{B i 1} \times{ }^{B} \boldsymbol{e}_{i}{ }^{B} \boldsymbol{p}_{B i 2} \times{ }^{B} \boldsymbol{e}_{i}\end{array}\right] \in \boldsymbol{R}^{3 \times 2}, \quad i=1,2$,

$\boldsymbol{W}_{G V 1}=\left[\begin{array}{cccc}{ }^{B} \boldsymbol{e}_{3} & { }^{B} \boldsymbol{e}_{3} & { }^{B} \boldsymbol{e}_{3} & { }^{B} \boldsymbol{e}_{3} \\ { }^{B} \boldsymbol{p}_{B 31} \times{ }^{B} \boldsymbol{e}_{3}{ }^{B} \boldsymbol{p}_{B 32} \times{ }^{B} \boldsymbol{e}_{3} & { }^{B} \boldsymbol{p}_{B 41} \times{ }^{B} \boldsymbol{e}_{3}{ }^{B} \boldsymbol{p}_{B 42} \times{ }^{B} \boldsymbol{e}_{3}\end{array}\right] \in \boldsymbol{R}^{3 \times 4}$

Introducing the coefficient matrix $\boldsymbol{\alpha}$, which represents the tension forces of the DAMs with VCMs under passive constraints, we obtain

$$
\left[\begin{array}{l}
\boldsymbol{T}_{31} \\
\boldsymbol{T}_{32} \\
\boldsymbol{T}_{41} \\
\boldsymbol{T}_{42}
\end{array}\right]=\boldsymbol{\alpha}\left[\begin{array}{l}
\boldsymbol{T}_{310} \\
\boldsymbol{T}_{320}
\end{array}\right], \quad \boldsymbol{\alpha}=\left[\begin{array}{cc}
\alpha_{31} & 0 \\
0 & \alpha_{32} \\
1-\alpha_{31} & 0 \\
0 & 1-\alpha_{32}
\end{array}\right] \in \boldsymbol{R}^{4 \times 2},
$$

where $\boldsymbol{T}_{3 i 0}, i=1,2$ are the independent tensions in the DAM with VCMs. Later, these tensions will be separated into two dependent wire tensions. $\alpha_{3 i}$ and $\left(1-\alpha_{3 i}\right)$ should be zero or positive.

Introducing the constraint matrix $C$, which relates the set of all the wire tensions to the set of all independent wire tensions as follows:

$$
\begin{aligned}
& \boldsymbol{T}=\boldsymbol{C}^{\mathbf{T}} \boldsymbol{T}_{C}, \\
& \boldsymbol{C}=\left[\begin{array}{cc}
\boldsymbol{E}_{4} & 0 \\
0 & \boldsymbol{\alpha}
\end{array}\right] \in \boldsymbol{R}^{8 \times 6}
\end{aligned}
$$

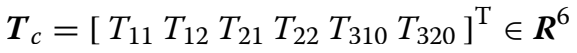

Substituting Eq. (30) into Eq. (27), the static force under the passive constraints imposed by the VCMs is given by

$$
\boldsymbol{F}_{G}=\boldsymbol{W}_{G C} \boldsymbol{T}_{C},
$$

where

$$
\boldsymbol{W}_{G C}=\left[\boldsymbol{W}_{G 1} \boldsymbol{W}_{G 2} \boldsymbol{W}_{G C 1}\right]^{\mathrm{T}} \in \boldsymbol{R}^{3 \times 6},
$$

with

$$
\boldsymbol{W}_{G C 1}=\left[\begin{array}{c}
{ }^{B} \boldsymbol{e}_{3} \\
\left\{\alpha_{31}{ }^{B} \boldsymbol{p}_{B 31}+\left(1-\alpha_{31}\right)^{B} \boldsymbol{p}_{B 41}\right\} \times{ }^{B} \boldsymbol{e}_{3} \quad\left\{\alpha_{31}{ }^{B} \boldsymbol{p}_{B 31}+\left(1-\alpha_{31}\right)^{B} \boldsymbol{p}_{B 41}\right\} \times{ }^{B} \boldsymbol{e}_{3}
\end{array}\right] \in \boldsymbol{R}^{3 \times 2} .
$$

In constrast, the producible global velocity, that is, the translational velocity $\dot{\boldsymbol{p}}$ in the global motion space, is determined by kinematical analysis as follows:

$$
\boldsymbol{v}_{G}=C_{P} \dot{\boldsymbol{p}},
$$

where, $\boldsymbol{v}_{G}=\left[\dot{\boldsymbol{p}}^{\mathrm{T}} \dot{\boldsymbol{\phi}}^{\mathrm{T}}\right]^{\mathrm{T}} \in \boldsymbol{R}^{3}, \dot{\boldsymbol{p}} \in \boldsymbol{R}^{2}$. The producible velocity matrix $C_{P}$, which relates the translational velocity $\dot{\boldsymbol{p}}$ and the global motion velocity $\boldsymbol{\nu}_{G}$ is defined as follows:

$$
\boldsymbol{C}_{P}=\left[\begin{array}{ll}
1 & 0 \\
0 & 1 \\
0 & 0
\end{array}\right] \in \boldsymbol{R}^{3 \times 2} .
$$

As the velocity relation is dual to the static force relation, the resultant force for global motion in the velocity producible space is given by:

$$
\boldsymbol{f}=\boldsymbol{C}_{P}^{\mathrm{T}} \boldsymbol{F}_{G},
$$

Substituting $\boldsymbol{F}_{G}$ in Eq. (31) into Eq. (34), the static force relation between $\boldsymbol{f}$ and $\boldsymbol{T}_{C}$ is obtained as:

$$
\boldsymbol{f}=\boldsymbol{C}_{P}^{\mathrm{T}} \boldsymbol{W}_{G} \boldsymbol{C} \boldsymbol{T}_{C} .
$$

Note that the moments in $\boldsymbol{F}_{G}$, which include the coefficients $\alpha_{3 i}$ will be disappeared.

The same result can be obtained through Eqs. (34), (27) and (30):

$$
\boldsymbol{f}=\boldsymbol{W}_{C V C} \boldsymbol{T}_{C},
$$

where $W_{C V C}$ is the matrix of resultant force on the top plate in the constraint space. It is derived as follows:

$$
W_{C V C}=C_{P}^{\mathrm{T}} \boldsymbol{W}_{G} \boldsymbol{C},
$$

In the general case, let us suppose that the RDWM includes $N_{D}$ DAMs without VCMs and $N_{V}$ DAMs with VCMs. Then we have $\boldsymbol{F}_{G}=\left[\boldsymbol{f}^{\mathrm{T}} \boldsymbol{n}^{\mathrm{T}}\right]^{\mathrm{T}} \in \boldsymbol{R}^{n}$, where $\boldsymbol{f} \in \boldsymbol{R}^{p}$ and $\boldsymbol{n} \in \boldsymbol{R}^{n-p}$, [here, $(n, p)=(2,1)$ in $1 \mathrm{D},(3,2)$ in $2 \mathrm{D}$ and $(6,3)$ in $3 \mathrm{D}] . \boldsymbol{F}_{G}$ is the resultant force vector that produce global motion, $\boldsymbol{T}=\left[T_{11} T_{12} \ldots T_{N_{D} 1} T_{N_{D} 2} T_{\left(N_{D}+1\right) 1} T_{\left(N_{D}+1\right) 2} T_{\left(N_{D}+2\right) 1}\right.$

$T_{\left(N_{D}+2\right) 2} \ldots T_{\left(N_{D}+2 N_{V}-1\right) 1} T_{\left(N_{D}+2 N_{V}-1\right) 2} T_{\left(N_{D}+2 N_{V}\right) 1}$ $\left.T_{\left(N_{D}+2 N_{V}\right) 2}\right]^{T} \in \boldsymbol{R}^{2\left(N_{D}+2 N_{V}\right)}$ is the wire tension vector. The matrix $W_{G}$ is given by Eq. (23):

$$
\begin{aligned}
\boldsymbol{W}_{G}= & {\left[\begin{array}{llllll}
\boldsymbol{W}_{G 1} & \ldots & \boldsymbol{W}_{G N_{D}} & \boldsymbol{W}_{G V 1} & \ldots & \boldsymbol{W}_{G V N_{V}}
\end{array}\right]^{\mathrm{T}} } \\
& \in \boldsymbol{R}^{n \times 2\left(N_{D}+2 N_{V}\right)},
\end{aligned}
$$


where the matrices $W_{G i}$ and $W_{G V m}$ are obtained from Eqs. (13) and (18).

Again introducing the coefficient matrix $\boldsymbol{\alpha}$, which represents the tension forces of the DAMs with VCMs under passive constraints, we obtain

$$
\begin{aligned}
& {\left[\begin{array}{c}
\boldsymbol{T}_{k 1} \\
\boldsymbol{T}_{k 2} \\
\boldsymbol{T}_{(k+1) 1} \\
\boldsymbol{T}_{(k+1) 2}
\end{array}\right]=\boldsymbol{\alpha}_{m}\left[\begin{array}{l}
\boldsymbol{T}_{k 10} \\
\boldsymbol{T}_{k 20}
\end{array}\right],} \\
& \boldsymbol{\alpha}_{m}=\left[\begin{array}{cc}
\alpha_{k 1} & 0 \\
0 & \alpha_{k 2} \\
1-\alpha_{k 1} & 0 \\
0 & 1-\alpha_{k 2}
\end{array}\right] \in \boldsymbol{R}^{4 \times 2}
\end{aligned}
$$

where $k=N_{D}+2 m-1 ; m=1, \ldots, N_{V}$.

Here, $\boldsymbol{T}_{k u 0}, u=1,2$ are the independent tensions in the DAM with VCMs, which later separate into two dependent wire tensions, $\boldsymbol{T}_{(k+u-1) 1}, \boldsymbol{T}_{(k+u-1) 2}$, and $\alpha_{k u}$ $\left(0 \leq \alpha_{k u} \leq 1, u=1,2\right)$ is the coefficient that distributes the wire tensions $\boldsymbol{T}_{k u 0}$ into two dependent wire tensions.

Introducing the constraint matrix $\boldsymbol{C}$, which relates the set of all the wire tensions to the set of all independent wire tensions as follows:

$$
\boldsymbol{T}=\boldsymbol{C}^{\mathbf{T}} \boldsymbol{T}_{C},
$$

where $\boldsymbol{T}_{c}=\left[T_{11} T_{12} \ldots T_{N_{D} 1} T_{N_{D} 2} T_{\left(N_{D}+1\right) 10} T_{\left(N_{D}+1\right) 20} T_{\left(N_{D}+3\right) 10}\right.$ $\left.T_{\left(N_{D}+1\right) 20} \ldots T_{\left(N_{D}+1 N_{V}-1\right) 10} T_{\left(N_{D}+2 N_{V}-1\right) 20}\right]^{T} \in \boldsymbol{R}^{2\left(N_{D} l N_{V}\right)}$ is the vector of independent wire tensions.

Here,

$\boldsymbol{C}=$ bdiag. $\left(\boldsymbol{E}_{2 N_{D}}, \boldsymbol{\alpha}_{1}, \ldots, \boldsymbol{\alpha}_{N_{V}}\right) \in \boldsymbol{R}^{2\left(N_{D}+2 N_{V}\right) \times 2\left(N_{D}+N_{V}\right)}$

$\boldsymbol{E}_{2 N_{D}} \in \boldsymbol{R}^{2 N_{D} \times 2 N_{D}}$ is the identity matrix.

Substituting Eq. (40) into (27), the static force relation under the passive constraints imposed by the VCMs is given by

$$
\boldsymbol{F}_{G}=\boldsymbol{W}_{G C} \boldsymbol{T}_{C},
$$

where

$$
\begin{aligned}
\boldsymbol{W}_{G C}= & {\left[\boldsymbol{W}_{G 1} \cdots \boldsymbol{W}_{G N_{D}} \boldsymbol{W}_{G C 1} \cdots \boldsymbol{W}_{G C N_{D}}\right]^{\mathrm{T}} } \\
& \in \boldsymbol{R}^{n \times 2\left(N_{D}+2 N_{V}\right)},
\end{aligned}
$$

here

$$
\begin{aligned}
& \boldsymbol{W}_{G i}= {\left[\begin{array}{cc}
{ }^{B} \boldsymbol{e}_{i} & { }^{B} \boldsymbol{e}_{i} \\
{ }^{B} \boldsymbol{p}_{B i 1} \times{ }^{B} \boldsymbol{e}_{i} & { }^{B} \boldsymbol{p}_{B i 2} \times{ }^{B} \boldsymbol{e}_{i}
\end{array}\right] } \\
& \in \boldsymbol{R}^{\mathrm{n} \times 2} i=1, \ldots N_{D}, \\
& \boldsymbol{W}_{G C m}= {\left[\begin{array}{c}
{ }^{B} \boldsymbol{e}_{k} \\
\left.\alpha_{k 1}{ }^{B} \boldsymbol{p}_{B k 1}+\left(1-\alpha_{k 1}\right)^{B} \boldsymbol{p}_{B(k+1) 1}\right\} \times{ }^{B} \boldsymbol{e}_{k}
\end{array}\right.} \\
& \in \boldsymbol{R}^{\mathrm{n} \times 2},\left(k=N_{D}+2 m-1 ; m=1, \ldots N_{V}\right) .
\end{aligned}
$$

Again, the producible global velocity (translational velocity $\dot{\boldsymbol{p}}$ in the global motion space) is derived from kinematical analysis:

$$
\boldsymbol{v}_{G}=C_{P} \dot{\boldsymbol{p}},
$$

where, $v_{G}=\left[\dot{\boldsymbol{p}}^{\mathrm{T}} \dot{\phi}^{\mathrm{T}}\right]^{\mathrm{T}} \in \boldsymbol{R}^{n}, \dot{\boldsymbol{p}} \in \boldsymbol{R}^{p}$. The producible velocity matrix $C_{P}$, which relates the translational velocity $\dot{\boldsymbol{p}}$ and the global motion velocity $\boldsymbol{\nu}_{G}$ is defined as follows:

$$
\boldsymbol{C}_{P}=\left[\begin{array}{c}
\boldsymbol{E}_{p \times p} \\
\boldsymbol{O}_{(n-p) \times p}
\end{array}\right] \in \boldsymbol{R}^{n \times p},
$$

$[(n, p)=(2,1)$ in $1 \mathrm{D},(3,2)$ in $2 \mathrm{D}$ and $(6,3)$ in $3 \mathrm{D}]$.

As the velocity relation is dual to the static force relation, the resultant force for global motion in the velocity producible space is given by:

$$
\boldsymbol{f}=\boldsymbol{C}_{P}^{\mathrm{T}} \boldsymbol{F}_{G},
$$

Substituting $\boldsymbol{F}_{G}$ in Eq. (42) into (45), the static force relation between $\boldsymbol{f}$ and $\boldsymbol{T}_{C}$ is obtained as:

$$
\boldsymbol{f}=\boldsymbol{C}_{P}^{\mathrm{T}} \boldsymbol{W}_{G} \boldsymbol{C} \boldsymbol{T}_{C},
$$

Note that the moments in $\boldsymbol{F}_{G}$, which include the coefficients $\alpha_{k u}$ will be disappeared.

The same result can be obtained through Eqs. (45), (27) and (40):

$$
\boldsymbol{f}=\boldsymbol{W}_{C V C} \boldsymbol{T}_{C},
$$

where $W_{C V C}$ is the matrix of resultant force on the top plate in the constraint space. It is derived as follows:

$$
W_{C V C}=C_{P}^{\mathrm{T}} \boldsymbol{W}_{G} \boldsymbol{C},
$$

Second, Eq. (36) or (47) will be used in SFA for judging whether the resultant force can be produced in any direction within the active constraint space $S_{A C}$. The procedure is described below.

C1) Find $\operatorname{rank}\left(\boldsymbol{W}_{C V C}\right)$.

C2) Find a vector $\boldsymbol{T}_{C}>\mathbf{0}$ that satisfies $\boldsymbol{W}_{C V C} \boldsymbol{T}_{C}=\mathbf{0}$.

The rank $\left(\boldsymbol{W}_{C V C}\right)$ in condition $\mathrm{C} 1$ defines the number of directions where the resultant forces are produced in the active constraint space $S_{A C}$. If $\operatorname{rank}\left(W_{C V C}\right)$ is full, the resultant forces can be produced in any direction in $S_{A C}$. In contrast, if $\operatorname{rank}\left(\boldsymbol{W}_{C V C}\right)$ is not full, the resultant force (and hence the velocity of the top plate) will be forbidden in one or more directions. In this case, the SFA fails and the RDWM candidate should be eliminated. Under

$\left.\left\{\alpha_{k 2}{ }^{B} \boldsymbol{p}_{B k 2}+\left(1-\alpha_{k 2}\right)^{B} \boldsymbol{p}_{B(k+1) 2}\right\} \times{ }^{B} \boldsymbol{e}_{k}\right]$ 
condition $\mathrm{C} 2$, the matrix $W_{C V C}$ is non-regular because the sizes of the wire tension vector in the constraint coordinate $\boldsymbol{T}_{C}$ and the resultant force vector in the active constraint space ${ }^{c} \boldsymbol{F}$ are mismatched. Consequently, the number of roots exceeds the number of equations. If the candidate RDWM configuration is feasible, we can find a positive value of the wire tension $\boldsymbol{T}_{C}$ satisfying $\boldsymbol{W}_{C V C} \boldsymbol{T}_{C}=\mathbf{0}$. If the RDWM candidate passes the SFA, the resultant force can be produced in any direction within the active constraint space.

The proposed procedure with three steps specify the necessary and sufficient conditions for judging the RDWM candidates, respectively. If a candidate RDWM satisfies the above procedure, it can generate the desired motions. Hence, the proposed judgment determines proper configurations of the RDWM.

\section{Numerical examples}

\section{Proper configuration of the 1D wire mechanism with one} VCM

The 1D wire mechanism with one VCM is shown in Fig. 6 . Here, the top plate under the gravity force will be suspended by one VCM. The gravity force $p=m g$ has any positive value which depends on the mass of the top plate $m$ under the acceleration of gravity $g$. For the purpose of simplifying, let's assume that the gravity force has the magnitude $p=1[\mathrm{~N}]$.

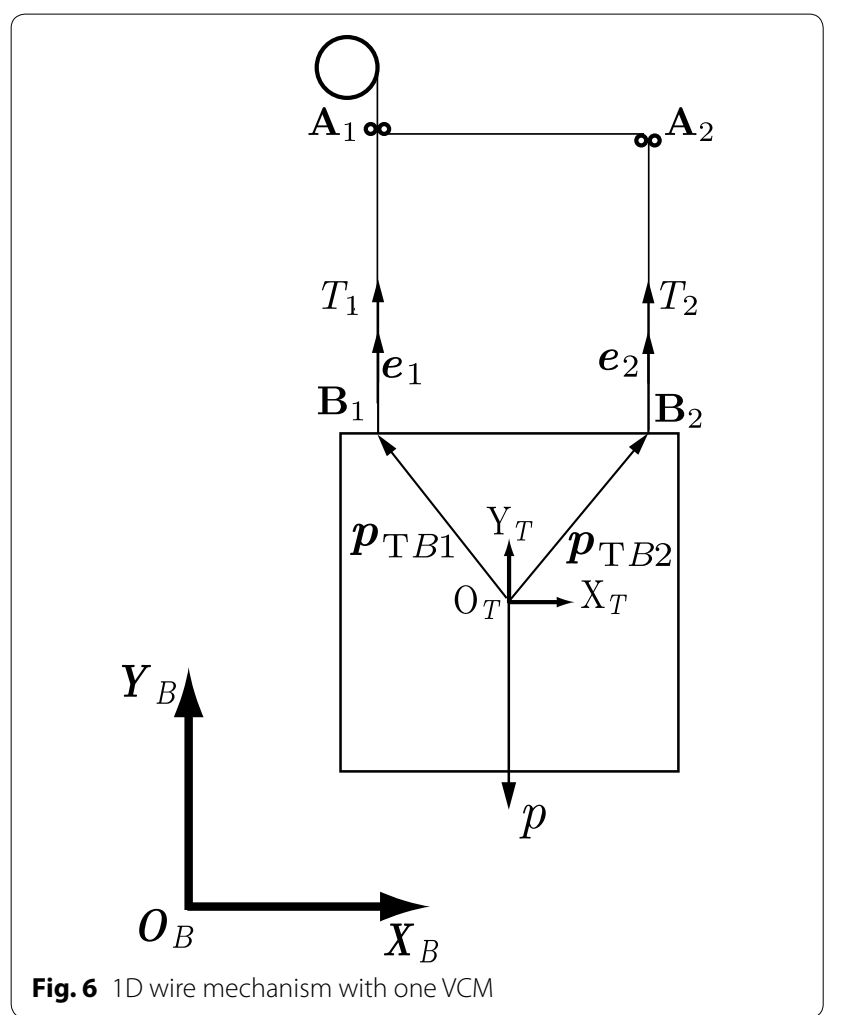

\section{Necessary condition check (step 1)}

In step 1, the matrix $W_{G}$ that contributes to the resultant force on the top plate is given by Eq. (23) with only one VCM contain two wires as follows:

$$
W_{G}=\left[\begin{array}{cc}
0 & 0 \\
1 & 1 \\
44 & 56
\end{array}\right] \in \boldsymbol{R}^{3 \times 2} .
$$

The first row of $W_{G}$ above has all zero elements so the top plate cannot move in $\mathrm{X}$ direction. Then, $W_{G}$ can be written again as follows:

$$
W_{G}=\left[\begin{array}{cc}
1 & 1 \\
44 & 56
\end{array}\right] \in \boldsymbol{R}^{2 \times 2} .
$$

For checking vector closure condition, the gravity force on the top plate is introduced. Then the matrix $\widetilde{W}_{G}$ contains the gravity component is shown as follows:

$$
\widetilde{W}=\left[\begin{array}{ccc}
1 & 1 & -1 \\
44 & 56 & -50
\end{array}\right] \in R^{2 \times 3} .
$$

Correspond to the matrix $\widetilde{W}_{G}$ is a vector $\widetilde{\boldsymbol{T}}=\left[T_{1} T_{2} p\right]^{\mathrm{T}} \in \boldsymbol{R}^{3}$ which contains the wire tensions $T_{1}, T_{2}$ and the gravity force $p$. It is easily seen that:

1. $\operatorname{rank}(\widetilde{\boldsymbol{W}})=2$.

2. The vector $\widetilde{\boldsymbol{T}}_{0}=\left[\begin{array}{lll}0.5 & 0.51\end{array}\right]^{\mathrm{T}} \in \boldsymbol{R}^{3}>\mathbf{0}$ satisfies $\widetilde{W} \widetilde{\boldsymbol{T}}_{0}=\mathbf{0}$.

Therefore, this 1D wire mechanism satisfies step 1 and the analysis proceeds to the step 2 as follows.

\section{KA (step 2)}

The matrices of contribution of VCM to the velocity of the top plate is given as follows:

$$
\boldsymbol{A}_{L}=\left[\begin{array}{cc}
-1 & 0 \\
1 & 0 \\
1 & 1 \\
-1 & 1
\end{array}\right] \in \boldsymbol{R}^{4 \times 2}, \quad \boldsymbol{b}_{L}=\left[\begin{array}{l}
1 \\
1 \\
0 \\
0
\end{array}\right] \in \boldsymbol{R}^{4}
$$

The matrices of top plate velocities $\boldsymbol{A}_{V}$ and $\boldsymbol{b}_{V}$ are given by Eqs. (20) and (21), respectively. The result are given below:

$$
\boldsymbol{A}_{V}=\left[\begin{array}{cc}
-1 & -44 \\
1 & 44 \\
0 & 12 \\
0 & -12
\end{array}\right] \in \boldsymbol{R}^{4 \times 2}, \quad \boldsymbol{b}_{V}=\left[\begin{array}{l}
1 \\
1 \\
0 \\
0
\end{array}\right] \in \boldsymbol{R}^{4}
$$

From the above $\boldsymbol{A}_{V}, \boldsymbol{b}_{V}$, we solve the convex sets mentioned in Eqs. (19) and (24). The vertex sets in matrix $\boldsymbol{A}$, the active constraint space $S_{A C}$ and passive constraint space $S_{P C}$ of the 1D wire mechanism are found by Eqs. (25) and (26). The result are shown below. 


$$
\begin{aligned}
& A=\left[\begin{array}{cc}
-1 & 1 \\
0 & 0
\end{array}\right] \in \boldsymbol{R}^{2 \times 2} \\
& S_{A C}=\mathcal{R}\left(\left[\begin{array}{l}
1 \\
0
\end{array}\right]\right), \quad S_{P C}=\mathcal{R}\left(\left[\begin{array}{l}
0 \\
1
\end{array}\right]\right) .
\end{aligned}
$$

Equation (55) shows that in this case, the active constraint space $S_{A C}$ produced by the top plate allows motion in the $\mathrm{Y}$ direction and the orientation is fixed around the Z-axis.

\section{SFA (step 3)}

The matrix $\widetilde{\boldsymbol{W}}_{C V C}$ that relates the wire tension vector in the constraint coordinate is derived from Eq. (48) then it is used for SFA. Because the wire tension in the general space is the same as the wire tension in the constraint space, the matrix $C$ is an identity matrix in this case. The contents of the matrix $\widetilde{\boldsymbol{W}}_{C V C}$ are given below:

$$
\widetilde{W}_{C V C}=\left[\begin{array}{lll}
1 & 1 & -1
\end{array}\right] \in \boldsymbol{R}^{1 \times 3}
$$

$\widetilde{W}_{C V C}$ contains some of the elements of matrix $\widetilde{W}_{G}$. We then have:

1. $\operatorname{rank}\left(\widetilde{\boldsymbol{W}}_{C V C}\right)=1$.

2. The vector $\widetilde{\boldsymbol{T}}_{0}=\left[\begin{array}{llll}0.5 & 0.5 & 1\end{array}\right]^{\mathrm{T}} \in \boldsymbol{R}^{3}>\mathbf{0}$ satisfies $\widetilde{\boldsymbol{W}}_{C V C} \widetilde{\boldsymbol{T}}_{0}=\mathbf{0}$.

The above analysis reveals that $\widetilde{W}_{C V C}$ is a full-ranked matrix. Moreover, there is a vector $\widetilde{\boldsymbol{T}}_{0}$ that satisfies $\widetilde{\boldsymbol{W}}_{C V C} \widetilde{\boldsymbol{T}}_{0}=\mathbf{0}$. Consequently, this wire mechanism satisfies step 3 and the resultant force can be produced in any direction within the active constraint space $\boldsymbol{S}_{A C}$.

Because it passes all three of the judgment steps, this wire mechanism is a proper configuration that achieves the desired active constraint space $S_{A C}$ in Y direction.

\section{Proper configuration of the planar RDWM with fixed orientation around the $\mathrm{Z}$-axis while maintaining translational motion in the $\mathrm{X}$ and $\mathrm{Y}$ directions}

Suppose we require a planar RDWM wherein the desired active constraint space $S_{A C}$ is the XOY plane and the passive constraint space $S_{P C}$ is the orientation around the Z-axis of the top plate. Various configurations of the planar RDWM are proposed and assessed by the judgment procedure developed in the previous section. All lengthbased parameters in this analysis are in $(\mathrm{cm})$.

\section{First configuration: planar RDWM with four DAMs}

The first configuration is a wire mechanism with four sets of DAMs, as shown in Fig. 3.

\section{Necessary condition check (step 1)}

In step 1 , the matrix $W_{G}$ that contributes to the resultant force on the top plate is given by Eq. (23) as follows:

$$
W_{G}=\frac{1}{5}\left[\begin{array}{cccccccc}
-4 & -4 & 4 & 4 & 0 & 0 & 0 & 0 \\
-3 & -3 & -3 & -3 & 5 & 5 & 5 & 5 \\
14 & -2 & 2 & -14 & 40 & 20 & -20 & -40
\end{array}\right] \in \boldsymbol{R}^{3 \times 8}
$$

It is easily seen that:

1. $\operatorname{rank}\left(\boldsymbol{W}_{G}\right)=3$.

2. The wire tension vector $\boldsymbol{T}_{0}=\left[\begin{array}{l}5 \\ 5\end{array} 5553333\right]^{\mathrm{T}}$ $\in \boldsymbol{R}^{8}>\mathbf{0}$ satisfies $\boldsymbol{W}_{G} \boldsymbol{T}_{0}=\mathbf{0}$.

Therefore, this planar RDWM candidate satisfies step 1 and the analysis proceeds to the step 2 as follows.

\section{KA (step 2)}

The matrix of total contribution of DAMs to the velocity of the top plate is given by Eq. (22):

$$
\boldsymbol{A}_{L}=\text { bdiag. }\left(\boldsymbol{A}_{L d 1}, \boldsymbol{A}_{L d 2}, \boldsymbol{A}_{L d 3}, \boldsymbol{A}_{L d 4}\right) \in \boldsymbol{R}^{16 \times 8} .
$$

Equation (11) presents the contents of $\boldsymbol{A}_{L d 1}, \boldsymbol{A}_{L d 2}, \boldsymbol{A}_{L d 3}$, $\boldsymbol{A}_{L d 4}$ and $\boldsymbol{b}_{L d 1}, \boldsymbol{b}_{L d 2}, \boldsymbol{b}_{L d 3}, \boldsymbol{b}_{L d 4}$. The matrices of top plate velocities $\boldsymbol{A}_{V}$ and $\boldsymbol{b}_{V}$ are given by Eqs. (20) and (21), respectively. The result are given below:

$A_{V}=\frac{1}{5}\left[\begin{array}{ccc}4 & 3 & -14 \\ -4 & -3 & 14 \\ 4 & 3 & 2 \\ -4 & -3 & -2 \\ -4 & 3 & -2 \\ 4 & -3 & 2 \\ -4 & 3 & 14 \\ 4 & -3 & -14 \\ 0 & -5 & -40 \\ 0 & 5 & 40 \\ 0 & -5 & -20 \\ 0 & 5 & 20 \\ 0 & -5 & 20 \\ 0 & 5 & -20 \\ 0 & -5 & 40 \\ 0 & 5 & -40\end{array}\right] \in \boldsymbol{R}^{16 \times 3}, \quad \boldsymbol{b}_{V}=\left[\begin{array}{l}1 \\ 1 \\ 1 \\ 1 \\ 1 \\ 1 \\ 1 \\ 1 \\ 1 \\ 1 \\ 1 \\ 1 \\ 1 \\ 1 \\ 1 \\ 1\end{array}\right] \in \boldsymbol{R}^{16}$.

From the above $\boldsymbol{A}_{V}, \boldsymbol{b}_{V}$, we solve the convex sets mentioned in Eqs. (19) and (24). The vertex sets in matrix $\boldsymbol{A}$, the active constraint space $S_{A C}$ and passive constraint space $S_{P C}$ of the planar RDWM with four sets of DAM are found by Eqs. (25) and (26). The result are shown below. 


$$
\begin{gathered}
\boldsymbol{A}=\left[\begin{array}{ccccc}
-1.25 & 1.25 & -1.1875 & -0.5 & 0.5 \\
0 & 0 & 0 & -1 & 1 \\
0 & 0 & -0.125 & 0 & 0
\end{array}\right. \\
\left.\begin{array}{ccccc}
1.1875 & 0.5 & -0.5 & -0.8125 & 0.8125 \\
0 & -1 & 1 & 0 & 0 \\
0.125 & 0 & 0 & 0.125 & -0.125
\end{array}\right] \in \boldsymbol{R}^{3 \times 10} \\
\boldsymbol{S}_{A C}=\mathcal{R}\left(\boldsymbol{E}_{3}\right), \quad \boldsymbol{S}_{P C}=\emptyset .
\end{gathered}
$$

Equation (61) shows that in this case, the active constraint space $S_{A C}$ produced by the top plate allows motion in the $\mathrm{X}$, and $\mathrm{Y}$ directions and the rotation around the $\mathrm{Z}$-axis.

\section{SFA (step 3)}

The above KA revealed that the active constraint space $S_{A C}$ is the whole motion space of the mechanism. Therefore, the matrix $W_{C V C}$ which relates the wire tension vector to the resultant force vector in the constraint coordinates is exactly the matrix $W_{G}$ in Eq. (57). Then, similar to step 1 , we easily observe that:

1. $\operatorname{rank}\left(\boldsymbol{W}_{C V C}\right)=3$.

2. The wire tension vector $\boldsymbol{T}_{C 0}=\left[\begin{array}{l}5 \\ 5\end{array} 553333\right]^{\mathrm{T}}$ $\in \boldsymbol{R}^{8}>\mathbf{0}$ satisfies $\boldsymbol{W}_{C V C} \boldsymbol{T}_{C 0}=\mathbf{0}$.

The matrix $W_{C V C}$ is full-ranked in the above analysis. Moreover, there is a vector $\boldsymbol{T}_{C 0}$ that satisfies $\boldsymbol{W}_{C V C} \boldsymbol{T}_{C 0}=\mathbf{0}$. Therefore, this RDWM candidate passes step 3 and the resultant force can be produced in any direction within the active constraint space $S_{A C \text {. }}$

As this configuration of planar RDWM does not contrain the velocity, velocities can be generated in any direction (step 2). Consequently, the contents of step 3 are identical to those of step 1.

Therefore, to find proper configurations of a RDWM without VCM, only step 1 is enough. However, not only step 1 but also steps 2, 3 are necessary for the case of RDWM with VCM as revealed next.

\section{Second configuration: planar RDWM with two DAMs and one DAM with a VCM}

We now propose a planar RDWM configured with two DAMs without VCMs and one DAM with a VCM (see Fig. 7). This configuration is identical to the planar RDWM with four DAMs in the last example, except that the top two DAMs are replaced by a DAM with a VCM.

\section{Necessary condition check (step 1)}

The matrix $W_{G}$ of this configuration is given by Eq. (57) and step 1 proceeds as described for the first configuration.

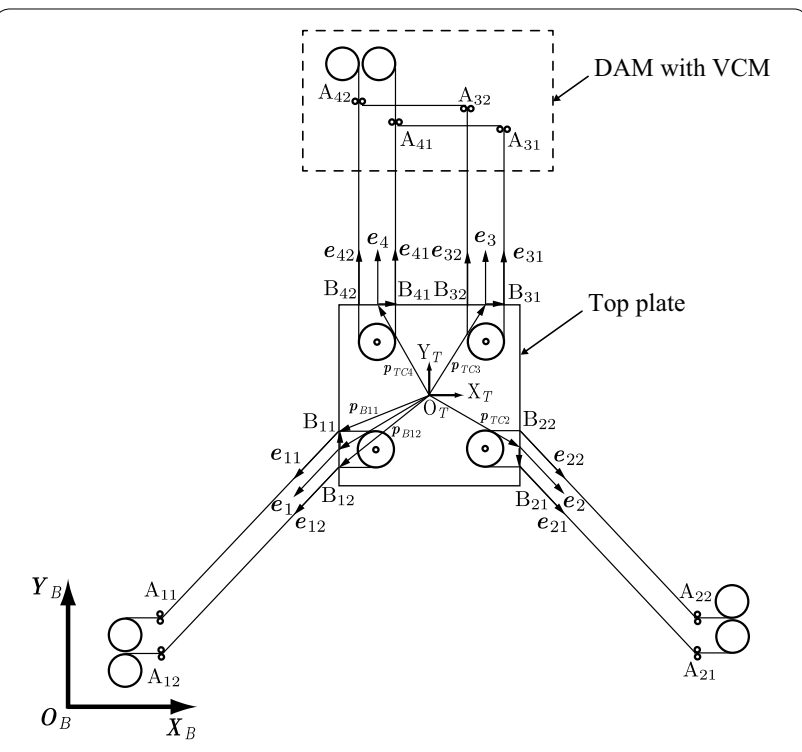

Fig. 7 Planar RDWM with two DAMs and one VCM

\section{KA (step 2)}

The matrix of total contributions of the DAMs and VCM to the top plate's velocity is given by Eq. (22):

$$
\boldsymbol{A}_{L}=\text { bdiag. }\left(\boldsymbol{A}_{L d 1}, \boldsymbol{A}_{L d 2}, \boldsymbol{A}_{L v}\right) \in \boldsymbol{R}^{16 \times 8} \text {. }
$$

Here the contents of $\boldsymbol{A}_{L d 1}, \boldsymbol{A}_{L d 2}, \boldsymbol{A}_{L v}$ and $\boldsymbol{b}_{L d 1}, \boldsymbol{b}_{L d 2}, \boldsymbol{b}_{L v}$ are shown in Eqs. (11) and (16). The matrices of top plate's velocities $\boldsymbol{A}_{V}, \boldsymbol{b}_{V}$ are obtained from Eqs. (20) and (21), and the results are shown below:

$A_{V}=\frac{1}{5}\left[\begin{array}{ccc}4 & 3 & -14 \\ -4 & -3 & 14 \\ 4 & 3 & 2 \\ -4 & -3 & -2 \\ -4 & 3 & -2 \\ 4 & -3 & 2 \\ -4 & 3 & 14 \\ 4 & -3 & -14 \\ 0 & -5 & -40 \\ 0 & 5 & 40 \\ 0 & 0 & 60 \\ 0 & 0 & -60 \\ 0 & -5 & -20 \\ 0 & 5 & 20 \\ 0 & 0 & 60 \\ 0 & 0 & -60\end{array}\right] \in \boldsymbol{R}^{16 \times 3}, \quad \boldsymbol{b}_{V}=\left[\begin{array}{l}1 \\ 1 \\ 1 \\ 1 \\ 1 \\ 1 \\ 1 \\ 1 \\ 1 \\ 1 \\ 0 \\ 0 \\ 1 \\ 1 \\ 0 \\ 0\end{array}\right] \in \boldsymbol{R}^{16}$

From the above $\boldsymbol{A}_{V}, \boldsymbol{b}_{V}$, we solve the convex sets mentioned in Eqs. (19) and (24). The vertex sets in matrix $\boldsymbol{A}$, the active constraint space $S_{A C}$ and the passive constraint space $S_{P C}$ of the planar RDWM with two DAMs and one DAM with a VCM are obtained by Eqs. (25) and (26) and are respectively given by 


$$
\begin{aligned}
A= & {\left[\begin{array}{cccccc}
-1.25 & 1.25 & -0.5 & 0.5 & 0.5 & -0.5 \\
0 & 0 & -1 & 1 & -1 & 1 \\
0 & 0 & 0 & 0 & 0 & 0
\end{array}\right] \in R^{3 \times 6}, } \\
& S_{A C}=\mathcal{R}\left(\left[\begin{array}{ll}
1 & 0 \\
0 & 1 \\
0 & 0
\end{array}\right]\right), \quad S_{P C}=\mathcal{R}\left(\left[\begin{array}{l}
0 \\
0 \\
1
\end{array}\right]\right) .
\end{aligned}
$$

Equation (65) indicates that the top plate can move in the $\mathrm{X}$ and $\mathrm{Y}$ directions but its orientation is fixed around the $\mathrm{Z}$-axis. Therefore, this configuration satisfies the step 2 and the analysis proceeds to step 3 .

\section{SFA (step 3)}

The matrix $\boldsymbol{W}_{C V C}$ that relates the wire tension vector in the constraint coordinate is derived from Eq. (48) then it is used for SFA. The contents of this matrix are given below:

$$
\boldsymbol{W}_{C V C}=\frac{1}{5}\left[\begin{array}{cccccc}
-4 & -4 & 4 & 4 & 0 & 0 \\
-3 & -3 & -3 & -3 & 5 & 5
\end{array}\right] \in \boldsymbol{R}^{2 \times 6}
$$

$W_{C V C}$ contains some of the elements of matrix $W_{G}$. We then have:

1. $\operatorname{rank}\left(\boldsymbol{W}_{C V C}\right)=2$.

2. The wire tension vector $\boldsymbol{T}_{C 0}=\left[\begin{array}{llllll}3 & 2 & 3 & 2 & 3 & 3\end{array}\right]^{\mathrm{T}} \in \boldsymbol{R}^{6}>\mathbf{0}$ satisfies $\boldsymbol{W}_{C V C} \boldsymbol{T}_{C 0}=\mathbf{0}$.

The above analysis reveals that $W_{C V C}$ is a full-ranked matrix. Moreover, there is a vector $\boldsymbol{T}_{C 0}$ that satisfies $\boldsymbol{W}_{C V C} \boldsymbol{T}_{C 0}=\mathbf{0}$. Consequently, this RDWM candidate satisfies step 3 and the resultant force can be produced in any direction within the active constraint space $\boldsymbol{S}_{A C}$.

Because it passes all three of the judgment steps, this planar RDWM candidate with two DAMs and one DAM with a VCM is a proper configuration that achieves the desired active constraint space $S_{A C}$ in X and Y directions. This configuration requires two fewer actuators than the planar RDWM with four DAMs.

\section{Third configuration: improper planar RDWM with two DAMs and one DAM with a VCM}

Figure 8 shows an improper planar RDWM with two DAMs and one DAM with a VCM. This configuration has the same modules as configuration 2 , but the module arrangement is inappropriate.

\section{Necessary condition check (step 1)}

In step 1 , the matrix $W_{G}$ of contributions to the resultant force exerted on the top plate is obtained by Eq. (23) as follows:

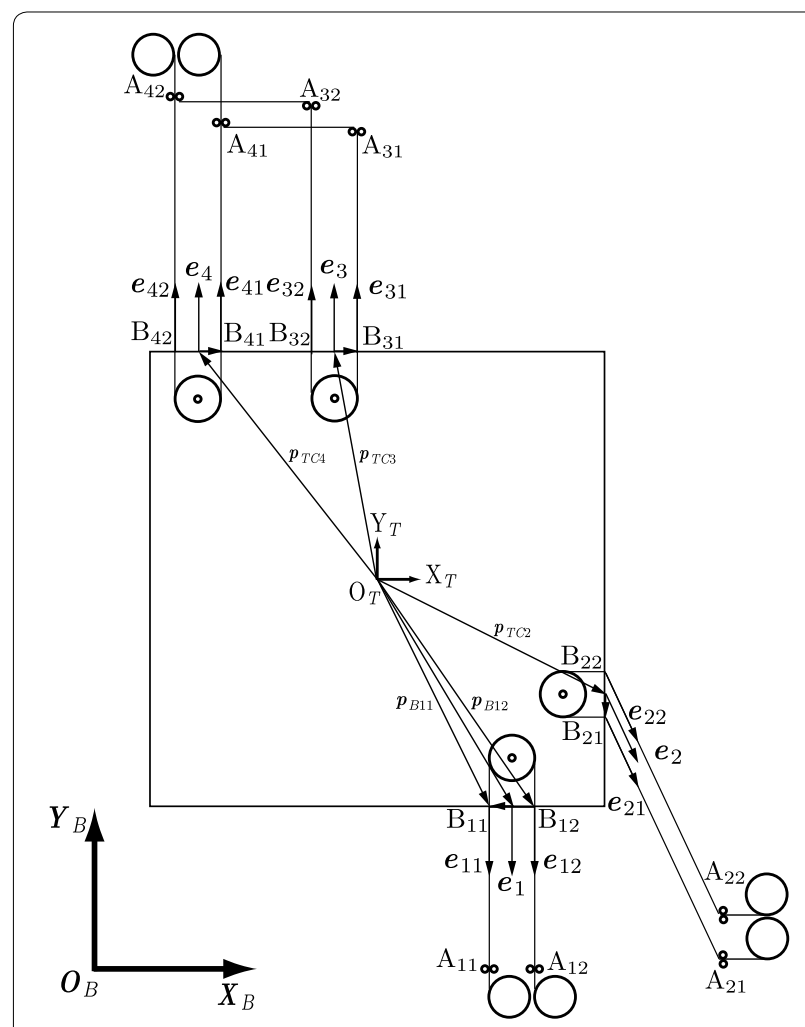

Fig. 8 Improper planar RDWM configuration with two DAMs and one VCM

It is easily determined that although $\operatorname{rank}\left(\boldsymbol{W}_{G}\right)=3$ (satisfying the necessary condition $\mathrm{C} 1$ ), this configuration fails $\mathrm{C} 2$. The resultant moments around the $\mathrm{Z}$-axis (third row of matrix $W_{G}$ ) are all negative, meaning that any positive wire tension vector will produce a clockwise moment. Therefore, the mechanism fails step 1, so the judgment procedure is terminated and this RDWM candidate is eliminated.

\section{Proper configuration of 3D RDWM with fixed orientations} around $\mathrm{X}$-, $\mathrm{Y}$-, and $\mathrm{Z}$-axes while maintaining translational motions in the $X, Y$ and $Z$ directions

Similar to the previous example, we now develop a $3 \mathrm{D}$ RDWM wherein the desired active constraint space $S_{A C}$ enables motions of the top plate in the $\mathrm{X}, \mathrm{Y}$ and $\mathrm{Z}$ directions, and the passive constraint space $S_{P C}$ prohibits orientation of the top plate around the three axes. We propose two 3D configurations and apply the judgment procedures in the previous section to determine whether the mechanisms can produce the desired active 
constrained motion space. As before, the unit of all length-related parameters is $(\mathrm{cm})$.

\section{First configuration: 3D RDWM with seven DAMs}

The first configuration is a wire mechanism using seven set of DAMs, as shown in Fig. 9.

\section{Necessary condition check (step 1)}

In step 1 , the matrix $W_{G}$ contributing to the resultant force exerted on the top plate is obtained by Eq. (23). It is easy to clarify that this 3D RDWM candidate satisfies the step 1 ; thus, the analysis proceeds to step 2 .

\section{KA (step 2)}

From the $\boldsymbol{A}_{V}, \boldsymbol{b}_{V}$ in Eq. (78) in Appendix 3, the convex set in Eqs. (19) and (24) can be solved. The results includes the vertex sets in matrix $A$ (sized $6 \times 172$ ). Then, the active constraint space $S_{A C}$, the passive constraint space $S_{P C}$ of the 3D RDWM with seven DAM sets are obtained by Eq. (26). The result is shown below:

$$
S_{A C}=\mathcal{R}\left(E_{6}\right), \quad S_{P C}=\emptyset .
$$

Equation (68) means that in this configuration, the active constraint space $S_{A C}$ produced by the top plate allows motions of the top plate in the $\mathrm{X}, \mathrm{Y}$, and $\mathrm{Z}$ directions and also rotations around all three axes.

\section{SFA (step 3)}

The above KA reveals that the active constraint space $\boldsymbol{S}_{A C}$ is the whole motion space of the mechanism. Therefore, the matrix $W_{C V C}$ which relates the wire tension vector to the resultant force vector in the constraint coordinates is exactly the matrix $W_{G}$. Then, similar to step 1 , we can

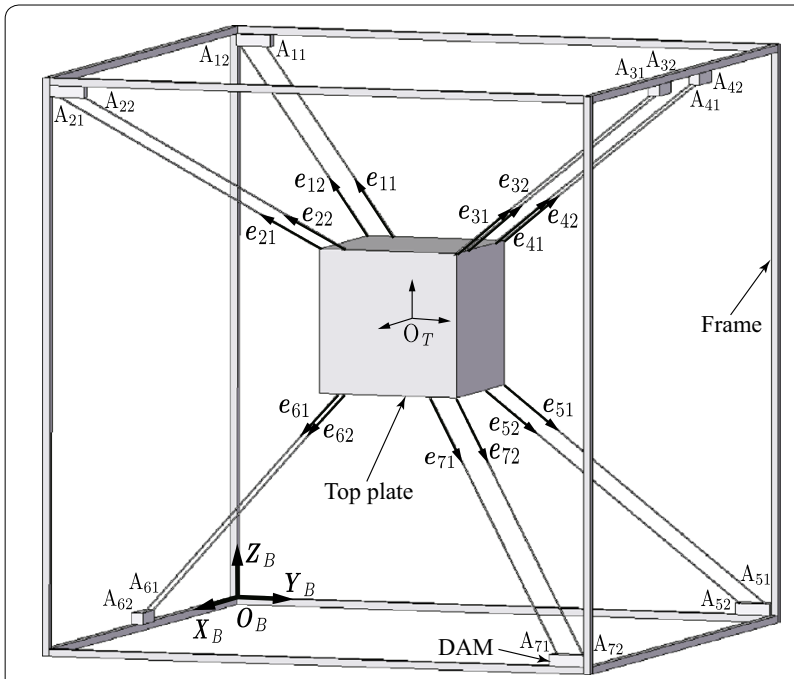

Fig. 9 3D RDWM with seven DAMs easily determine that this RDWM candidate satisfies step 3 and generates a resultant force in any direction within the active constraint space $S_{A C}$.

\section{Second configuration: 3D RDWM with four sets of DAMs with VCMs}

Finally, we configure a 3D RDWM with four sets of DAMs each with a VCM (see Fig. 10).

\section{Necessary condition check (step 1)}

Step 1 analyzes the following matrix $W_{G}$ of contributions to the resultant force on the top plate. This matrix, obtained from Eq. (23) is given by:

$$
\begin{gathered}
W_{G}=\frac{1}{3}\left[\begin{array}{cccccccc}
-1 & -1 & -1 & -1 & 2 & 2 & 2 & 2 \\
-2 & -2 & -2 & -2 & 2 & 2 & 2 & 2 \\
-2 & -2 & -2 & -2 & 1 & 1 & 1 & 1 \\
34 & 26 & 10 & 2 & -24 & -12 & 12 & 24 \\
-24 & -12 & 12 & 24 & 7 & -1 & -17 & -25 \\
7 & -1 & -17 & -25 & 34 & 26 & 10 & 2 \\
1 & 1 & 1 & 1 & -2 & -2 & -2 & -2 \\
2 & 2 & 2 & 2 & -2 & -2 & -2 & -2 \\
-2 & -2 & -2 & -2 & 1 & 1 & 1 & 1 \\
-34 & -26 & -10 & -2 & 24 & 12 & -12 & -24 \\
24 & 12 & -12 & -24 & -7 & 1 & 17 & 25 \\
7 & -1 & -17 & -25 & 34 & 26 & 10 & 2
\end{array}\right] \in R^{6 \times 16}
\end{gathered}
$$

It is easily observed that

1. $\operatorname{rank}\left(\boldsymbol{W}_{G}\right)=6$.

2. The wire tension vector $\boldsymbol{T}_{0}=[5535351010$ $707055353510107070]^{\mathrm{T}} \in \boldsymbol{R}^{16}>\mathbf{0} \quad$ satisfies $\boldsymbol{W}_{G} \boldsymbol{T}_{0}=\mathbf{0}$.

Therefore, this 3D RDWM candidate satisfies step 1 and the analysis proceeds to step 2 .

\section{KA (step 2)}

As the previous examples, step 2 derives the active constraint space $S_{A C}$ and the passive constraint space $S_{P C}$ of the 3D RDWM using four sets of DAMs with VCMs by Eq. (26). The result is shown below:

$$
\boldsymbol{S}_{A C}=\mathcal{R}\left(\left[\begin{array}{l}
\boldsymbol{E}_{3} \\
\boldsymbol{O}_{3}
\end{array}\right]\right), \quad \boldsymbol{S}_{P C}=\mathcal{R}\left(\left[\begin{array}{l}
\boldsymbol{O}_{3} \\
\boldsymbol{E}_{3}
\end{array}\right]\right) .
$$

Equation (70) shows that the configured 3D RDWM can move in the $\mathrm{X}, \mathrm{Y}$ and $\mathrm{Z}$ directions but cannot rotate around the $\mathrm{X}$-, $\mathrm{Y}$ - and $\mathrm{Z}$-axes. Therefore, this configuration satisfies step 2 and the analysis proceeds to step 3 .

\section{SFA (step 3)}

The matrix $W_{C V C}$ which relates the wire tension vector to the resultant force vector in the constrained coordinate is 


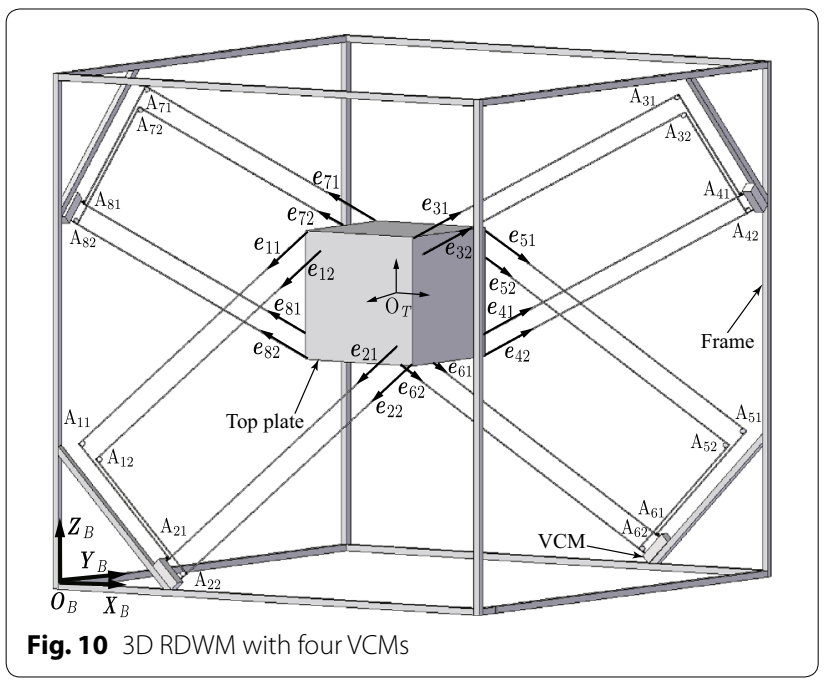

derived from Eq. (48) and subjected to SFA. The content of this matrix are shown below:

$$
W_{C V C}=\frac{1}{3}\left[\begin{array}{cccccccc}
-1 & -1 & 2 & 2 & 1 & 1 & -2 & -2 \\
-2 & -2 & 2 & 2 & 2 & 2 & -2 & -2 \\
-2 & -2 & 1 & 1 & -2 & -2 & 1 & 1
\end{array}\right] \in \boldsymbol{R}^{3 \times 8},
$$

The matrix $W_{C V C}$ contains some of the elements of matrix $W_{G}$. We then have:

1. $\operatorname{rank}\left(\boldsymbol{W}_{C V C}\right)=3$.

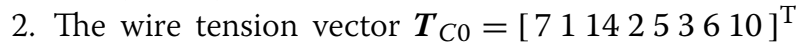
$\in \boldsymbol{R}^{8}>\mathbf{0}$ satisfying $\boldsymbol{W}_{C V C} \boldsymbol{T}_{C 0}=\mathbf{0}$.

The above analysis shows that $W_{C V C}$ is a full-ranked matrix. Moreover, as there exists a vector $\boldsymbol{T}_{C 0}$ satisfying $\boldsymbol{W}_{C V C} \boldsymbol{T}_{C 0}=\mathbf{0}$, this RDWM candidate satisfies step 3 and the resultant force can be produced in any direction within the active constraint space $S_{A C}$. Because it passes all three steps of the judgment procedure, the four sets of DAMs with VCMs form a proper 3D RDWM configuration that generates the desired active constraint space $S_{A C}$ in the X, $\mathrm{Y}$ and $\mathrm{Z}$ directions. This configuration requires six fewer actuators than the 3D RDWM with seven DAMs.

\section{Conclusions}

This study addresses a method to reduce the required number of actuators for redundant drive wire mechanism (RDWM)-wire mechanism that can produce fast and fine motions by introducing velocity constraint modules (VCMs) into its configuration. The concept of the research is producing the top plate with fast-translational motion for moving through large working space while fixing its orientations by using VCMs. Instead of changing the orientation of the whole top plate, the orientation of the end point of top plate can be changed largely to produce fine motion by local mechanism. This study also proposed a procedure that judges whether an RDWM configuration is appropriate for generating the target motions. The main results are summarized below:

1. A procedure for judging candidate RDWMs was introduced. Similar to a conventional wire mechanism, an RDWM must generate a resultant force in any direction over the whole motion space. The VCMs must then constrain the orientation of the top plate by judging the producible velocity space of the top plate. Because the top plate moves in a restricted region of the space, it must produce a resultant force in any direction within this space. Each of these requirements is tested in one step of the proposed three-steps judgment procedure. The validity of the judgment procedure was confirmed in numerical examples of 1D, 2D and 3D configurations.

2. The examples verified that VCMs effectively reduce the number of actuators while maintaining the orientation of the top plate. Moreover, in the 3D RDWM configured with four sets of DAMs with VCMs, the sets of tensions in the four directions generate translational forces in any direction on the top plate, enabling global translational motions in 3D space.

The findings of this study will contribute to the structure design of a RDWM with VCMs. An investigation on vibration from the wire elasticity and a vibration suppression method will be considered in future work.

\section{Authors' contributions}

TNL carried out the study on the proposed judgment procedure, performed analysis of the numerical examples, drafted the manuscript, and revised it. HD helped to establish the proposed judgment procedure, carried out the study on it, provided technical advice, and helped to draft and revise the manuscript. KN designed the study, conceived the proposed judgment procedure, provided technical advice, and helped to draft and revise the manuscript. All authors read and approved the final manuscript.

\section{Acknowledgements}

This work was supported by The Ministry of Education, Culture, Sports, Science and Technology of Japan, Grant-in-Aid for Scientific Research (B), No. 24300206.

Competing interests

The authors declare that we have no competing interests. 


\section{Appendix 1: List of notations and symbols}

See Table 1.

Table 1 List of notations and symbols

\begin{tabular}{llll}
\hline No. & $\begin{array}{l}\text { Notations or } \\
\text { symbols }\end{array}$ & Description & Locations \\
\hline 1. & $\times$ & The cross product of two vectors in $R^{3}$.For two vectors in $R^{2}$, the result of this & Eqs. (13), (18) \\
& & $\mathbf{a} \times \mathbf{b}=\left|\begin{array}{ll}a_{1} & a_{2} \\
b_{1} & b_{2}\end{array}\right|=a_{1} b_{2}-a_{2} b_{1}$ & Details of step 1, Eqs. (13), (18) \\
& & & Number of global motion DoFs of the top plate
\end{tabular}

\section{Appendix 2: Derivation of the wire matrix} Position of wire end points on the top plate w.r.t the top plate coordinate ${ }^{T} \mathbf{p}_{B i j}$

First, the vectors ${ }^{T} \boldsymbol{p}_{T C i},{ }^{T} \boldsymbol{p}_{T D i}$ and radii $R_{i}$ are defined. The positions of the wire end points on the top plate w.r.t the top plate coordinate ${ }^{T} \boldsymbol{p}_{B i j}$ are then given by the following equation:

$$
\left\{\begin{array}{l}
{ }^{T} \boldsymbol{p}_{B i 1}={ }^{T} \boldsymbol{p}_{T C i}+{ }^{T} \boldsymbol{p}_{T D i}, \\
{ }^{T} \boldsymbol{p}_{B i 2}={ }^{T} \boldsymbol{P}_{T C i}-{ }^{T} \boldsymbol{p}_{T D i} .
\end{array}\right.
$$

Position of wire end points on the top plate w.r.t the base coordinate ${ }^{B} p_{B i j}$

This is given by the following equation:

$$
{ }^{B} \boldsymbol{p}_{B i j}={ }^{B} \boldsymbol{p}_{T}+{ }^{B} \boldsymbol{R}_{T}{ }^{\mathrm{T}} \boldsymbol{p}_{B i j},
$$

where ${ }^{B} \boldsymbol{p}_{T}$ is the initial position of the top plate and ${ }^{B} \boldsymbol{R}_{T}$ is the rotational matrix from the base coordinate relative to the top coordinate.
Calculating the wire vectors ${ }^{B} \mathrm{e}_{i j}$

The wire vectors are computed by the following equation:

$$
{ }^{B} \boldsymbol{e}_{i j}=\frac{{ }^{B} \boldsymbol{p}_{A i j}-{ }^{B} \boldsymbol{p}_{B i j}}{\left\|\boldsymbol{p}_{A i j}-{ }^{B} \boldsymbol{p}_{B i j}\right\|},
$$

where, ${ }^{B} \boldsymbol{p}_{A i j}=\left[\begin{array}{lll}{ }^{B} x_{A i j} & { }^{B} y_{A i j} & { }^{B} z_{A i j}\end{array}\right]^{\mathrm{T}}$ are the positions of the wire end points on the frame w.r.t the base coordinate and ${ }^{B} \boldsymbol{p}_{B i j}=\left[\begin{array}{lll}{ }^{B} x_{B i j} & { }^{B} y_{B i j} & { }^{B} z_{B i j}\end{array}\right]^{\mathrm{T}}$ represents the positions of the wire end points on the top plate w.r.t the base coordinate. The symbol $\|$.$\| in Eq. (74) denotes the Euclidean norm of a vector.$

\section{Derivation of the wire matrix}

The whole wire matrix of the RDWM is defined as

$$
\boldsymbol{W}=\left[\begin{array}{l}
\boldsymbol{W}_{G} \\
\boldsymbol{W}_{L}
\end{array}\right] \in \boldsymbol{R}^{\left(n+N_{D}+2 N_{V}\right) \times 2\left(N_{D}+2 N_{V}\right)},
$$

where, the matrix $W$ contains the matrix $W_{G}$ of contributions to the resultant force on the top plate. $W_{G}$ is given by Eq. (23) and the matrix $W_{L}$ of contributions to the local moments of the local pulleys is given by:

$$
\boldsymbol{W}_{L}=\left[\begin{array}{ccccccc}
R_{1} & -R_{1} & 0 & 0 & \ldots & 0 & 0 \\
0 & 0 & R_{2} & -R_{2} & \ldots & 0 & 0 \\
\vdots & \vdots & \vdots & \vdots & \vdots & \vdots & \vdots \\
0 & 0 & 0 & 0 & \ldots & R_{N} & -R_{N}
\end{array}\right] \in \boldsymbol{R}^{\left(N_{D}+2 N_{V}\right) \times 2\left(N_{D}+2 N_{V}\right)}
$$




\section{Appendix 3: Parameters determinations in each configuration}

1D wire mechanism with one VCM

Positions of wire end points on the top plate w.r.t the top plate coordinate

$$
{ }^{T} \boldsymbol{p}_{B 1}=\left[\begin{array}{ll}
-6 & 10
\end{array}\right]^{\mathrm{T}}, \quad{ }^{T} \boldsymbol{p}_{B 2}=\left[\begin{array}{ll}
6 & 10
\end{array}\right]^{\mathrm{T}},
$$

Set of positions ${ }^{B} p_{T}$ and orientations ${ }^{B} R_{T}$ of the top plate

$$
{ }^{B} \boldsymbol{p}_{T}=\left[\begin{array}{ll}
50 & 50
\end{array}\right]^{\mathrm{T}}, \quad{ }^{B} \boldsymbol{R}_{T}=\left[\begin{array}{ll}
1 & 0 \\
0 & 1
\end{array}\right] \in \boldsymbol{R}^{2 \times 2} .
$$

Positions of wire end points on the top plate

From Eq. (73):

$$
{ }^{B} \boldsymbol{p}_{B 1}=\left[\begin{array}{ll}
44 & 60
\end{array}\right]^{\mathrm{T}}, \quad{ }^{B} \boldsymbol{p}_{B 2}=\left[\begin{array}{ll}
56 & 60
\end{array}\right]^{\mathrm{T}}
$$

Position of wire end points on the frame

The following positions of the wire end points on the frame are shown as follows:

$$
{ }^{B} \boldsymbol{p}_{A 1}=\left[\begin{array}{ll}
44 & 90
\end{array}\right]^{\mathrm{T}}, \quad{ }^{B} \boldsymbol{p}_{A 2}=\left[\begin{array}{ll}
56 & 90
\end{array}\right]^{\mathrm{T}} .
$$

Calculating the wire vectors ${ }^{B} e_{i}$

The wire vectors ${ }^{B} \boldsymbol{e}_{i}$ were calculated by Eq. (74).

$$
{ }^{B} \boldsymbol{e}_{1}={ }^{B} \boldsymbol{e}_{2}=\left[\begin{array}{ll}
0 & 1
\end{array}\right]^{\mathrm{T}}
$$

Planar RDWM with four DAMs

Defining ${ }^{T} p_{T C i}, R_{i}{ }^{T} p_{T D i}$ and ${ }^{T} p_{B i j}$

$$
\begin{aligned}
{ }^{T} \boldsymbol{p}_{T C 1} & =\left[\begin{array}{ll}
-10 & -6
\end{array}\right]^{\mathrm{T}}, & { }^{T} \boldsymbol{p}_{T C 2} & =\left[\begin{array}{ll}
10 & -6
\end{array}\right]^{\mathrm{T}}, \\
{ }^{T} \boldsymbol{p}_{T C 3} & =\left[\begin{array}{ll}
6 & 10
\end{array}\right]^{\mathrm{T}}, & { }^{T} \boldsymbol{p}_{T C 4} & =\left[\begin{array}{ll}
-6 & 10
\end{array}\right]^{\mathrm{T}} .
\end{aligned}
$$

The pulley radii are $R_{1}=R_{2}=R_{3}=R_{4}=2$.

$$
\begin{aligned}
& { }^{T} \boldsymbol{p}_{T D 1}=\left[\begin{array}{ll}
0 & 2
\end{array}\right]^{\mathrm{T}}, \quad{ }^{T} \boldsymbol{p}_{T D 2}=\left[\begin{array}{ll}
0 & -2
\end{array}\right]^{\mathrm{T}}, \\
& { }^{T} \boldsymbol{p}_{T D 3}=\left[\begin{array}{ll}
2 & 0
\end{array}\right]^{\mathrm{T}}, \quad{ }^{T} \boldsymbol{p}_{T D 4}=\left[\begin{array}{ll}
2 & 0
\end{array}\right]^{\mathrm{T}} \text {. }
\end{aligned}
$$

Positions of wire end points on the top plate w.r.t the top plate coordinate

From Eq. (72):

$$
\begin{array}{rlrl}
{ }^{T} \boldsymbol{p}_{B 11} & =\left[\begin{array}{ll}
-10 & -4
\end{array}\right]^{\mathrm{T}}, & { }^{T} \boldsymbol{p}_{B 12}=\left[\begin{array}{ll}
-10 & -8
\end{array}\right]^{\mathrm{T}}, \\
{ }^{T} \boldsymbol{p}_{B 21} & =\left[\begin{array}{ll}
10 & -8
\end{array}\right]^{\mathrm{T}}, & { }^{T} \boldsymbol{p}_{B 22} & =\left[\begin{array}{ll}
10 & -4
\end{array}\right]^{\mathrm{T}}, \\
{ }^{T} \boldsymbol{p}_{B 31} & =\left[\begin{array}{ll}
8 & 10
\end{array}\right]^{\mathrm{T}}, & & { }^{T} \boldsymbol{p}_{B 32}=\left[\begin{array}{ll}
4 & 10
\end{array}\right]^{\mathrm{T}}, \\
{ }^{T} \boldsymbol{p}_{B 41} & =\left[\begin{array}{ll}
-4 & 10
\end{array}\right]^{\mathrm{T}}, & { }^{T} \boldsymbol{p}_{B 42} & =\left[\begin{array}{ll}
-8 & 10
\end{array}\right]^{\mathrm{T}} .
\end{array}
$$

Set of positions ${ }^{B} p_{T}$ and orientations ${ }^{B} R_{T}$ of the top plate

$$
{ }^{B} \boldsymbol{p}_{T}=\left[\begin{array}{ll}
50 & 50
\end{array}\right]^{\mathrm{T}}, \quad{ }^{B} \boldsymbol{R}_{T}=\left[\begin{array}{ll}
1 & 0 \\
0 & 1
\end{array}\right] \in \boldsymbol{R}^{2 \times 2} .
$$

Positions of wire end points on the top plate From Eq. (73):

$$
\begin{array}{rlrl}
{ }^{B} \boldsymbol{p}_{B 11} & =\left[\begin{array}{ll}
40 & 46
\end{array}\right]^{\mathrm{T}}, & { }^{B} \boldsymbol{p}_{B 12} & =\left[\begin{array}{ll}
40 & 42
\end{array}\right]^{\mathrm{T}}, \\
{ }^{B} \boldsymbol{p}_{B 21} & =\left[\begin{array}{ll}
60 & 42
\end{array}\right]^{\mathrm{T}}, & { }^{B} \boldsymbol{p}_{B 22} & =\left[\begin{array}{ll}
60 & 46
\end{array}\right]^{\mathrm{T}}, \\
{ }^{B} \boldsymbol{p}_{B 31} & =\left[\begin{array}{ll}
58 & 60
\end{array}\right]^{\mathrm{T}}, & { }^{B} \boldsymbol{p}_{B 32}=\left[\begin{array}{ll}
54 & 60
\end{array}\right]^{\mathrm{T}}, \\
{ }^{B} \boldsymbol{p}_{B 41} & =\left[\begin{array}{ll}
46 & 60
\end{array}\right]^{\mathrm{T}}, & { }^{B} \boldsymbol{p}_{B 42} & =\left[\begin{array}{ll}
42 & 60
\end{array}\right]^{\mathrm{T}} .
\end{array}
$$

Position of wire end points on the frame

The following positions of the wire end points on the frame were ensured by appropriately arranging the DAMs on the frame:

$$
\begin{aligned}
{ }^{B} \boldsymbol{p}_{A 11} & =\left[\begin{array}{ll}
10 & 23.5
\end{array}\right]^{\mathrm{T}}, & { }^{B} \boldsymbol{p}_{A 12} & =\left[\begin{array}{ll}
10 & 19.5
\end{array}\right]^{\mathrm{T}}, \\
{ }^{B} \boldsymbol{p}_{A 21} & =\left[\begin{array}{ll}
90 & 19.5
\end{array}\right]^{\mathrm{T}}, & { }^{B} \boldsymbol{p}_{A 22} & =\left[\begin{array}{ll}
90 & 23.5
\end{array}\right]^{\mathrm{T}}, \\
{ }^{B} \boldsymbol{p}_{A 31} & =\left[\begin{array}{ll}
58 & 90
\end{array}\right]^{\mathrm{T}}, & { }^{B} \boldsymbol{p}_{A 32} & =\left[\begin{array}{ll}
54 & 90
\end{array}\right]^{\mathrm{T}}, \\
{ }^{B} \boldsymbol{p}_{A 41} & =\left[\begin{array}{ll}
46 & 90
\end{array}\right]^{\mathrm{T}}, & { }^{B} \boldsymbol{p}_{A 42} & =\left[\begin{array}{ll}
42 & 90
\end{array}\right]^{\mathrm{T}} .
\end{aligned}
$$

Calculating the wire vectors ${ }^{B} e_{i j}$

The wire vectors ${ }^{B} \boldsymbol{e}_{i j}$ were calculated by Eq. (74).

$$
\begin{aligned}
{ }^{B} \boldsymbol{e}_{11} & ={ }^{B} \boldsymbol{e}_{12}=\left[\begin{array}{ll}
-4 / 5 & -3 / 5
\end{array}\right]^{\mathrm{T}}, \\
{ }^{B} \boldsymbol{e}_{21} & ={ }^{B} \boldsymbol{e}_{22}=\left[\begin{array}{ll}
4 / 5 & -3 / 5
\end{array}{ }^{\mathrm{T}},\right. \\
{ }^{B} \boldsymbol{e}_{31} & ={ }^{B} \boldsymbol{e}_{32}=\left[\begin{array}{ll}
0 & 1
\end{array}\right]^{\mathrm{T}}, \\
{ }^{B} \boldsymbol{e}_{41} & ={ }^{B} \boldsymbol{e}_{42}=\left[\begin{array}{ll}
0 & 1
\end{array}\right]^{\mathrm{T}} .
\end{aligned}
$$

Planar RDWM with two DAMs and one VCM

The values of the parameters ${ }^{T} \boldsymbol{p}_{T C i}, R_{i},{ }^{T} \boldsymbol{p}_{T D i},{ }^{T} \boldsymbol{p}_{B i},{ }^{B} \boldsymbol{p}_{T}$, ${ }^{B} \boldsymbol{R}_{T}$ and ${ }^{B} \boldsymbol{p}_{B i j}$ were those assigned to the planar RDWM with four DAMs.

\section{Positions of wire end points on the frame}

The following positions of the wire end points on the frame were ensured by appropriately arranging the DAMs and VCMs on the frame:

$$
\begin{aligned}
{ }^{B} \boldsymbol{p}_{A 11} & =\left[\begin{array}{ll}
10 & 23.5
\end{array}\right]^{\mathrm{T}}, & { }^{B} \boldsymbol{p}_{A 12} & =\left[\begin{array}{ll}
10 & 19.5
\end{array}\right]^{\mathrm{T}}, \\
{ }^{B} \boldsymbol{p}_{A 21} & =\left[\begin{array}{ll}
90 & 19.5
\end{array}\right]^{\mathrm{T}}, & { }^{B} \boldsymbol{p}_{A 22} & =\left[\begin{array}{ll}
90 & 23.5
\end{array}\right]^{\mathrm{T}}, \\
{ }^{B} \boldsymbol{p}_{A 31} & =\left[\begin{array}{ll}
58 & 85
\end{array}\right]^{\mathrm{T}}, & { }^{B} \boldsymbol{p}_{A 32} & =\left[\begin{array}{ll}
54 & 90
\end{array}\right]^{\mathrm{T}}, \\
{ }^{B} \boldsymbol{p}_{A 41} & =\left[\begin{array}{ll}
46 & 85
\end{array}\right]^{\mathrm{T}}, & { }^{B} \boldsymbol{p}_{A 42} & =\left[\begin{array}{ll}
42 & 90
\end{array}\right]^{\mathrm{T}} .
\end{aligned}
$$

Calculating the wire vectors ${ }^{B} e_{i j}$

The wire vectors ${ }^{B} \boldsymbol{e}_{i j}$ calculated by Eq. (74), were identical to those of the planar RDWM with four DAMs.

Improper planar RDWM with two DAMs and one VCM Defining ${ }^{T} p_{T C i}, R_{i}{ }^{T} p_{T D i}$ and ${ }^{T} p_{B i j}$

$$
\begin{aligned}
{ }^{T} \boldsymbol{p}_{T C 1} & =\left[\begin{array}{ll}
16 & -20
\end{array}\right]^{\mathrm{T}}, & { }^{T} \boldsymbol{p}_{T C 2} & =\left[\begin{array}{ll}
20 & -10
\end{array}\right]^{\mathrm{T}}, \\
{ }^{T} \boldsymbol{p}_{T C 3} & =\left[\begin{array}{ll}
-4 & 20
\end{array}\right]^{\mathrm{T}}, & { }^{T} \boldsymbol{p}_{T C 4} & =\left[\begin{array}{ll}
-16 & 20
\end{array}\right]^{\mathrm{T}} .
\end{aligned}
$$


The pulley radii are: $R_{1}=R_{2}=R_{3}=R_{4}=2$.

$$
\begin{aligned}
{ }^{T} \boldsymbol{p}_{T D 1} & =\left[\begin{array}{ll}
-2 & 0
\end{array}\right]^{\mathrm{T}}, & { }^{T} \boldsymbol{p}_{T D 2} & =\left[\begin{array}{ll}
0 & -2
\end{array}\right]^{\mathrm{T}}, \\
{ }^{T} \boldsymbol{p}_{T D 3} & =\left[\begin{array}{ll}
2 & 0
\end{array}\right]^{\mathrm{T}}, & { }^{T} \boldsymbol{p}_{T D 4} & =\left[\begin{array}{ll}
2 & 0
\end{array}\right]^{\mathrm{T}} .
\end{aligned}
$$

The position of the wire end points on the top plate w.r.t the top plate coordinate were calculated by Eq. (72):

$$
\begin{aligned}
{ }^{T} \boldsymbol{p}_{B 11} & =\left[\begin{array}{ll}
14 & -20
\end{array}\right]^{\mathrm{T}}, & { }^{T} \boldsymbol{p}_{B 12} & =\left[\begin{array}{ll}
18 & -20
\end{array}\right]^{\mathrm{T}}, \\
{ }^{T} \boldsymbol{p}_{B 21} & =\left[\begin{array}{ll}
20 & -12
\end{array}\right]^{\mathrm{T}}, & { }^{T} \boldsymbol{p}_{B 22} & =\left[\begin{array}{ll}
20 & -8
\end{array}\right]^{\mathrm{T}}, \\
{ }^{T} \boldsymbol{p}_{B 31} & =\left[\begin{array}{ll}
-2 & 20
\end{array}{ }^{\mathrm{T}},\right. & { }^{T} \boldsymbol{p}_{B 32} & =\left[\begin{array}{ll}
-6 & 20
\end{array}\right]^{\mathrm{T}}, \\
{ }^{T} \boldsymbol{p}_{B 41} & =\left[\begin{array}{ll}
-14 & 20
\end{array}\right]^{\mathrm{T}}, & { }^{T} \boldsymbol{p}_{B 42} & =\left[\begin{array}{ll}
-18 & 20
\end{array}\right]^{\mathrm{T}} .
\end{aligned}
$$

Set of positions ${ }^{B} p_{T}$ and orientations ${ }^{B} R_{T}$ of the top plate

$$
{ }^{B} \boldsymbol{p}_{T}=\left[\begin{array}{ll}
50 & 50
\end{array}\right]^{\mathrm{T}},{ }^{B} \boldsymbol{R}_{T}=\left[\begin{array}{ll}
1 & 0 \\
0 & 1
\end{array}\right] \in \boldsymbol{R}^{2 \times 2} .
$$

\section{Positions of wire end points on the top plate}

From Eq. (73):

$$
\begin{array}{rlrl}
{ }^{B} \boldsymbol{p}_{B 11} & =\left[\begin{array}{ll}
64 & 30
\end{array}\right]^{\mathrm{T}}, & { }^{B} \boldsymbol{p}_{B 12}=\left[\begin{array}{ll}
68 & 30
\end{array}\right]^{\mathrm{T}}, \\
{ }^{B} \boldsymbol{p}_{B 21} & =\left[\begin{array}{ll}
70 & 38
\end{array}\right]^{\mathrm{T}}, & { }^{B} \boldsymbol{p}_{B 22} & =\left[\begin{array}{ll}
70 & 42
\end{array}\right]^{\mathrm{T}}, \\
{ }^{B} \boldsymbol{p}_{B 31} & =\left[\begin{array}{ll}
48 & 70
\end{array}\right]^{\mathrm{T}}, & { }^{B} \boldsymbol{p}_{B 32} & =\left[\begin{array}{ll}
44 & 70
\end{array}\right]^{\mathrm{T}}, \\
{ }^{B} \boldsymbol{p}_{B 41} & =\left[\begin{array}{lll}
36 & 70
\end{array}\right]^{\mathrm{T}}, & { }^{B} \boldsymbol{p}_{B 42} & =\left[\begin{array}{ll}
32 & 70
\end{array}\right]^{\mathrm{T}} .
\end{array}
$$

\section{Positions of wire end points on the frame}

The positions of the wire end points on the frame were ensured by appropriately arranging the DAMs and VCMs on the frame:

$$
\begin{aligned}
& { }^{B} \boldsymbol{p}_{A 11}=\left[\begin{array}{ll}
64 & 0
\end{array}\right]^{\mathrm{T}}, \quad{ }^{B} \boldsymbol{p}_{A 12}=\left[\begin{array}{ll}
68 & 0
\end{array}\right]^{\mathrm{T}}, \\
& { }^{B} \boldsymbol{p}_{A 21}=\left[\begin{array}{ll}
118 & 2
\end{array}\right]^{\mathrm{T}}, \quad{ }^{B} \boldsymbol{p}_{A 22}=\left[\begin{array}{ll}
118 & 6
\end{array}\right]^{\mathrm{T}} \text {, } \\
& { }^{B} \boldsymbol{p}_{A 31}=\left[\begin{array}{ll}
48 & 95
\end{array}\right]^{\mathrm{T}}, \quad{ }^{B} \boldsymbol{p}_{A 32}=\left[\begin{array}{ll}
44 & 100
\end{array}\right]^{\mathrm{T}} \text {, } \\
& { }^{B} \boldsymbol{p}_{A 41}=\left[\begin{array}{ll}
36 & 95
\end{array}\right]^{\mathrm{T}}, \quad{ }^{B} \boldsymbol{p}_{A 42}=\left[\begin{array}{ll}
32 & 100
\end{array}\right]^{\mathrm{T}} .
\end{aligned}
$$

Calculating the wire vectors ${ }^{B} e_{i j}$

The wire vectors ${ }^{B} \boldsymbol{e}_{i j}$ calculated by Eq. (74) are shown below:

$$
\begin{aligned}
{ }^{B} \boldsymbol{e}_{11} & ={ }^{B} \boldsymbol{e}_{12}=\left[\begin{array}{ll}
0 & -1
\end{array}\right]^{\mathrm{T}}, \\
{ }^{B} \boldsymbol{e}_{21} & ={ }^{B} \boldsymbol{e}_{22}=\left[\begin{array}{ll}
4 / 5 & -3 / 5
\end{array}\right]^{\mathrm{T}}, \\
{ }^{B} \boldsymbol{e}_{31} & ={ }^{B} \boldsymbol{e}_{32}=\left[\begin{array}{ll}
0 & 1
\end{array}\right]^{\mathrm{T}}, \\
{ }^{B} \boldsymbol{e}_{41} & ={ }^{B} \boldsymbol{e}_{42}=\left[\begin{array}{ll}
0 & 1
\end{array}\right]^{\mathrm{T}} .
\end{aligned}
$$

\section{D RDWM with seven DAMs}

\section{Defining ${ }^{T} p_{T C i}, R_{i},{ }^{T} p_{T D i}$ and ${ }^{T} p_{B i j}$}

$$
\begin{array}{rlrl}
{ }^{T} \boldsymbol{p}_{T C 1} & =\left[\begin{array}{lll}
-10 & -6 & 10
\end{array}\right]^{\mathrm{T}}, & { }^{T} \boldsymbol{p}_{T C 2}=\left[\begin{array}{lll}
10 & -6 & 10
\end{array}\right]^{\mathrm{T}}, \\
{ }^{T} \boldsymbol{p}_{T C 3} & =\left[\begin{array}{lll}
6 & 10 & 10
\end{array}\right]^{\mathrm{T}}, & { }^{T} \boldsymbol{p}_{T C 4}=\left[\begin{array}{lll}
-6 & 10 & 10
\end{array}\right]^{\mathrm{T}}, \\
{ }^{T} \boldsymbol{p}_{T C 5} & =\left[\begin{array}{lll}
-10 & 6 & -10
\end{array}\right]^{\mathrm{T}}, & { }^{T} \boldsymbol{p}_{T C 6}=\left[\begin{array}{lll}
0 & -10 & -10
\end{array}\right]^{\mathrm{T}}, \\
{ }^{T} \boldsymbol{p}_{T C 7} & =\left[\begin{array}{lll}
10 & 6 & -10
\end{array}\right]^{\mathrm{T}} .
\end{array}
$$

The pulley radii are: $R_{1}=R_{2}=R_{3}=R_{4}=R_{5}$ $=R_{6}=R_{7}=2$.

$$
\begin{aligned}
{ }^{T} \boldsymbol{p}_{T D 1} & =\left[\begin{array}{lll}
0 & 2 & 0
\end{array}\right]^{\mathrm{T}}, \quad{ }^{T} \boldsymbol{p}_{T D 2}=\left[\begin{array}{lll}
0 & -2 & 0
\end{array}\right]^{\mathrm{T}}, \\
{ }^{T} \boldsymbol{p}_{T D 3} & =\left[\begin{array}{lll}
2 & 0 & 0
\end{array}\right]^{\mathrm{T}}, \quad{ }^{T} \boldsymbol{p}_{T D 4}=\left[\begin{array}{lll}
2 & 0 & 0
\end{array}\right]^{\mathrm{T}}, \\
{ }^{T} \boldsymbol{p}_{T D 5} & =\left[\begin{array}{lll}
0 & 2 & 0
\end{array}\right]^{\mathrm{T}}, \quad{ }^{T} \boldsymbol{p}_{T D 6}=\left[\begin{array}{lll}
-2 & 0 & 0
\end{array}\right]^{\mathrm{T}}, \\
{ }^{T} \boldsymbol{p}_{T D 7} & =\left[\begin{array}{lll}
0 & -2 & 0
\end{array}\right]^{\mathrm{T}} .
\end{aligned}
$$

The positions of the wire end points on the top plate w.r.t the top plate coordinate were calculated by Eq. (72):

$$
\begin{array}{rlrl}
{ }^{T} \boldsymbol{p}_{B 11} & =\left[\begin{array}{lll}
-10 & -4 & 10
\end{array}\right]^{\mathrm{T}}, & { }^{T} \boldsymbol{p}_{B 12} & =\left[\begin{array}{lll}
-10 & -8 & 10
\end{array}\right]^{\mathrm{T}}, \\
{ }^{T} \boldsymbol{p}_{B 21} & =\left[\begin{array}{lll}
10 & -8 & 10
\end{array}\right]^{\mathrm{T}}, & & { }^{T} \boldsymbol{p}_{B 22}=\left[\begin{array}{lll}
10 & -4 & 10
\end{array}\right]^{\mathrm{T}}, \\
{ }^{T} \boldsymbol{p}_{B 31} & =\left[\begin{array}{lll}
8 & 10 & 10
\end{array}\right]^{\mathrm{T}}, & { }^{T} \boldsymbol{p}_{B 32}=\left[\begin{array}{lll}
4 & 10 & 10
\end{array}\right]^{\mathrm{T}}, \\
{ }^{T} \boldsymbol{p}_{B 41} & =\left[\begin{array}{lll}
-4 & 10 & 10
\end{array}\right]^{\mathrm{T}}, & { }^{T} \boldsymbol{p}_{B 42}=\left[\begin{array}{lll}
-8 & 10 & 10
\end{array}\right]^{\mathrm{T}}, \\
{ }^{T} \boldsymbol{p}_{B 51} & =\left[\begin{array}{lll}
-10 & 8 & -10
\end{array}\right]^{\mathrm{T}}, & { }^{T} \boldsymbol{p}_{B 52}=\left[\begin{array}{lll}
-10 & 4 & -10
\end{array}\right]^{\mathrm{T}}, \\
{ }^{T} \boldsymbol{p}_{B 61} & =\left[\begin{array}{llll}
-2 & -10 & -10
\end{array}\right]^{\mathrm{T}},{ }^{T} \boldsymbol{p}_{B 62}=\left[\begin{array}{lll}
2 & -10 & -10
\end{array}\right]^{\mathrm{T}}, \\
{ }^{T} \boldsymbol{p}_{B 71} & =\left[\begin{array}{llll}
10 & 4 & -10
\end{array}\right]^{\mathrm{T}}, & { }^{T} \boldsymbol{p}_{B 72} & =\left[\begin{array}{lll}
10 & 8 & -10
\end{array}\right]^{\mathrm{T}},
\end{array}
$$

Sets of position ${ }^{B} p_{T}$ and orientations ${ }^{B} R_{T}$ of the top plate

$$
{ }^{B} \boldsymbol{p}_{T}=\left[\begin{array}{lll}
50 & 50 & 50
\end{array}\right]^{\mathrm{T}},{ }^{B} \boldsymbol{R}_{T}=\left[\begin{array}{ccc}
1 & 0 & 0 \\
0 & 1 & 0 \\
0 & 0 & 1
\end{array}\right] \in \boldsymbol{R}^{3 \times 3} .
$$

\section{Positions of wire end points on the top plate}

From Eq. (73):

$$
\begin{aligned}
& { }^{B} \boldsymbol{p}_{B 11}=\left[\begin{array}{lll}
40 & 46 & 60
\end{array}\right]^{\mathrm{T}}, \quad{ }^{B} \boldsymbol{p}_{B 12}=\left[\begin{array}{lll}
40 & 42 & 60
\end{array}\right]^{\mathrm{T}}, \\
& { }^{B} \boldsymbol{p}_{B 21}=\left[\begin{array}{lll}
60 & 42 & 60
\end{array}\right]^{\mathrm{T}}, \quad{ }^{B} \boldsymbol{p}_{B 22}=\left[\begin{array}{lll}
60 & 46 & 60
\end{array}\right]^{\mathrm{T}} \text {, } \\
& { }^{B} \boldsymbol{p}_{B 31}=\left[\begin{array}{lll}
58 & 60 & 60
\end{array}\right]^{\mathrm{T}}, \quad{ }^{B} \boldsymbol{p}_{B 32}=\left[\begin{array}{lll}
54 & 60 & 60
\end{array}\right]^{\mathrm{T}} \text {, } \\
& { }^{B} \boldsymbol{p}_{B 41}=\left[\begin{array}{lll}
46 & 60 & 60
\end{array}\right]^{\mathrm{T}}, \quad{ }^{B} \boldsymbol{p}_{B 42}=\left[\begin{array}{lll}
42 & 60 & 60
\end{array}\right]^{\mathrm{T}} \text {, } \\
& { }^{B} \boldsymbol{p}_{B 51}=\left[\begin{array}{lll}
40 & 58 & 40
\end{array}\right]^{\mathrm{T}}, \quad{ }^{B} \boldsymbol{p}_{B 52}=\left[\begin{array}{lll}
40 & 54 & 40
\end{array}\right]^{\mathrm{T}} \text {, } \\
& { }^{B} \boldsymbol{p}_{B 61}=\left[\begin{array}{lll}
48 & 40 & 40
\end{array}\right]^{\mathrm{T}}, \quad{ }^{B} \boldsymbol{p}_{B 62}=\left[\begin{array}{lll}
52 & 40 & 40
\end{array}\right]^{\mathrm{T}} \text {, } \\
& { }^{B} \boldsymbol{p}_{B 71}=\left[\begin{array}{lll}
60 & 54 & 40
\end{array}\right]^{\mathrm{T}}, \quad{ }^{B} \boldsymbol{p}_{B 72}=\left[\begin{array}{lll}
60 & 58 & 40
\end{array}\right]^{\mathrm{T}} \text {. }
\end{aligned}
$$

\section{Positions of wire end points on the frame}

The positions of the wire end points on the frame were ensured by appropriately arranging the DAMs on the frame:

$$
\begin{aligned}
{ }^{B} \boldsymbol{p}_{A 11} & =\left[\begin{array}{lll}
10 & 46 & 100
\end{array}\right]^{\mathrm{T}}, & { }^{B} \boldsymbol{p}_{A 12} & =\left[\begin{array}{lll}
10 & 42 & 100
\end{array}\right]^{\mathrm{T}}, \\
{ }^{B} \boldsymbol{p}_{A 21} & =\left[\begin{array}{lll}
90 & 42 & 100
\end{array}\right]^{\mathrm{T}}, & { }^{B} \boldsymbol{p}_{A 22} & =\left[\begin{array}{llll}
90 & 46 & 100
\end{array}\right]^{\mathrm{T}}, \\
{ }^{B} \boldsymbol{p}_{A 31} & =\left[\begin{array}{llll}
58 & 90 & 100
\end{array}\right]^{\mathrm{T}}, & { }^{B} \boldsymbol{p}_{A 32} & =\left[\begin{array}{llll}
54 & 90 & 100
\end{array}\right]^{\mathrm{T}}, \\
{ }^{B} \boldsymbol{p}_{A 41} & =\left[\begin{array}{llll}
46 & 90 & 100
\end{array}\right]^{\mathrm{T}}, & { }^{B} \boldsymbol{p}_{A 42} & =\left[\begin{array}{llll}
42 & 90 & 100
\end{array}\right]^{\mathrm{T}}, \\
{ }^{B} \boldsymbol{p}_{A 51} & =\left[\begin{array}{lll}
10 & 58 & 0
\end{array}\right]^{\mathrm{T}}, & { }^{B} \boldsymbol{p}_{A 52} & =\left[\begin{array}{llll}
10 & 54 & 0
\end{array}\right]^{\mathrm{T}}, \\
{ }^{B} \boldsymbol{p}_{A 61} & =\left[\begin{array}{lll}
48 & 10 & 0
\end{array}\right]^{\mathrm{T}}, & { }^{B} \boldsymbol{p}_{A 62} & =\left[\begin{array}{llll}
52 & 10 & 0
\end{array}\right]^{\mathrm{T}}, \\
{ }^{B} \boldsymbol{p}_{A 71} & =\left[\begin{array}{lll}
90 & 54 & 0
\end{array}\right]^{\mathrm{T}}, & { }^{B} \boldsymbol{p}_{A 72} & =\left[\begin{array}{llll}
90 & 58 & 0
\end{array}\right]^{\mathrm{T}} .
\end{aligned}
$$


Calculating the wire vectors ${ }^{B} e_{i j}$

The wire vectors ${ }^{B} \boldsymbol{e}_{i j}$ were calculated by Eq. (74) and are listed below:

$$
\begin{aligned}
{ }^{B} \boldsymbol{e}_{21} & ={ }^{B} \boldsymbol{e}_{22}=\left[\begin{array}{lll}
-3 / 5 & 0 & 4 / 5
\end{array}\right]^{\mathrm{T}}, \\
{ }^{B} \boldsymbol{e}_{31} & ={ }^{B} \boldsymbol{e}_{32}=\left[\begin{array}{lll}
3 / 5 & 0 & 4 / 5
\end{array}\right]^{\mathrm{T}}, \\
{ }^{B} \boldsymbol{e}_{41} & ={ }^{B} \boldsymbol{e}_{42}=\left[\begin{array}{lll}
0 & 3 / 5 & 4 / 5
\end{array}\right]^{\mathrm{T}}, \\
{ }^{B} \boldsymbol{e}_{11} & ={ }^{B} \boldsymbol{e}_{12}=\left[\begin{array}{lll}
0 & 3 / 5 & 4 / 5
\end{array}\right]^{\mathrm{T}}, \\
{ }^{B} \boldsymbol{e}_{51} & ={ }^{B} \boldsymbol{e}_{52}=\left[\begin{array}{lll}
-3 / 5 & 0 & -4 / 5
\end{array}\right]^{\mathrm{T}}, \\
{ }^{B} \boldsymbol{e}_{61} & ={ }^{B} \boldsymbol{e}_{62}=\left[\begin{array}{lll}
0 & -3 / 5 & -4 / 5
\end{array}\right]^{\mathrm{T}}, \\
{ }^{B} \boldsymbol{e}_{71} & ={ }^{B} \boldsymbol{e}_{72}=\left[\begin{array}{lll}
3 / 5 & 0 & -4 / 5
\end{array}\right]^{\mathrm{T}} .
\end{aligned}
$$

KA (step 2)

From Eq. (22), the matrix of total VCM contributions to the velocity of the top plate is given by:

$$
\begin{aligned}
& \boldsymbol{A}_{L}=\operatorname{bdiag} .\left(\boldsymbol{A}_{L d 1}, \boldsymbol{A}_{L d 2}, \boldsymbol{A}_{L d 3},\right. \\
&\left.\boldsymbol{A}_{L d 4}, \boldsymbol{A}_{L d 5}, \boldsymbol{A}_{L d 6}, \boldsymbol{A}_{L d 7}\right) \in \boldsymbol{R}^{28 \times 14} .
\end{aligned}
$$

The contents of $\boldsymbol{A}_{L d i}$ and $\boldsymbol{b}_{L d i}$ are shown in Eq. (11). The matrices of the top plate's velocity $\boldsymbol{A}_{V}$ and $\boldsymbol{b}_{V}$ were then obtained by Eqs. (20) and (21). The result shown below:
3D RDWM with four VCMs

Defining ${ }^{T} p_{T C i}, R_{i}{ }^{T} p_{T D i}$, and ${ }^{T} p_{B i j}$

$$
\begin{aligned}
& { }^{T} \boldsymbol{p}_{T C 1}=\left[\begin{array}{lll}
-6 & -9 & 6
\end{array}\right]^{\mathrm{T}}, \quad{ }^{T} \boldsymbol{p}_{T C 2}=\left[\begin{array}{lll}
6 & -9 & -6
\end{array}\right]^{\mathrm{T}} \text {, } \\
& { }^{T} \boldsymbol{p}_{T C 3}=\left[\begin{array}{lll}
9 & -6 & 6
\end{array}\right]^{\mathrm{T}}, \quad{ }^{T} \boldsymbol{p}_{T C 4}=\left[\begin{array}{lll}
9 & 6 & -6
\end{array}\right]^{\mathrm{T}}, \\
& { }^{T} \boldsymbol{p}_{T C 5}=\left[\begin{array}{lll}
6 & 9 & 6
\end{array}\right]^{\mathrm{T}}, \quad{ }^{T} \boldsymbol{p}_{T C 6}=\left[\begin{array}{lll}
-6 & 9 & -6
\end{array}\right]^{\mathrm{T}}, \\
& { }^{T} \boldsymbol{p}_{T C 7}=\left[\begin{array}{lll}
-9 & 6 & 6
\end{array}\right]^{\mathrm{T}}, \quad{ }^{T} \boldsymbol{p}_{T C 8}=\left[\begin{array}{lll}
-9 & -6 & -6
\end{array}\right]^{\mathrm{T}} \text {. }
\end{aligned}
$$

The pulley radii are given by $R_{1}=R_{2}=R_{3}=R_{4}=R_{5}=$ $R_{6}=R_{7}=R_{8}=2 \sqrt{2}$.

$$
\begin{aligned}
& { }^{T} \boldsymbol{p}_{T D 1}=\left[\begin{array}{lll}
-2 & 0 & 2
\end{array}\right]^{\mathrm{T}}, \quad{ }^{T} \boldsymbol{p}_{T D 2}=\left[\begin{array}{lll}
-2 & 0 & 2
\end{array}\right]^{\mathrm{T}}, \\
& { }^{T} \boldsymbol{p}_{T D 3}=\left[\begin{array}{lll}
0 & -2 & 2
\end{array}\right]^{\mathrm{T}}, \quad{ }^{T} \boldsymbol{p}_{T D 4}=\left[\begin{array}{lll}
0 & -2 & 2
\end{array}\right]^{\mathrm{T}} \text {, } \\
& { }^{T} \boldsymbol{p}_{T D 5}=\left[\begin{array}{lll}
2 & 0 & 2
\end{array}\right]^{\mathrm{T}}, \quad{ }^{T} \boldsymbol{p}_{T D 6}=\left[\begin{array}{lll}
2 & 0 & 2
\end{array}\right]^{\mathrm{T}}, \\
& { }^{T} \boldsymbol{p}_{T D 7}=\left[\begin{array}{lll}
0 & 2 & 2
\end{array}\right]^{\mathrm{T}}, \quad{ }^{T} \boldsymbol{p}_{T D 8}=\left[\begin{array}{lll}
0 & 2 & 2
\end{array}\right]^{\mathrm{T}} .
\end{aligned}
$$

The positions of the wire end points on the top plate w.r.t the top plate coordinate were calculated by Eq. (72):

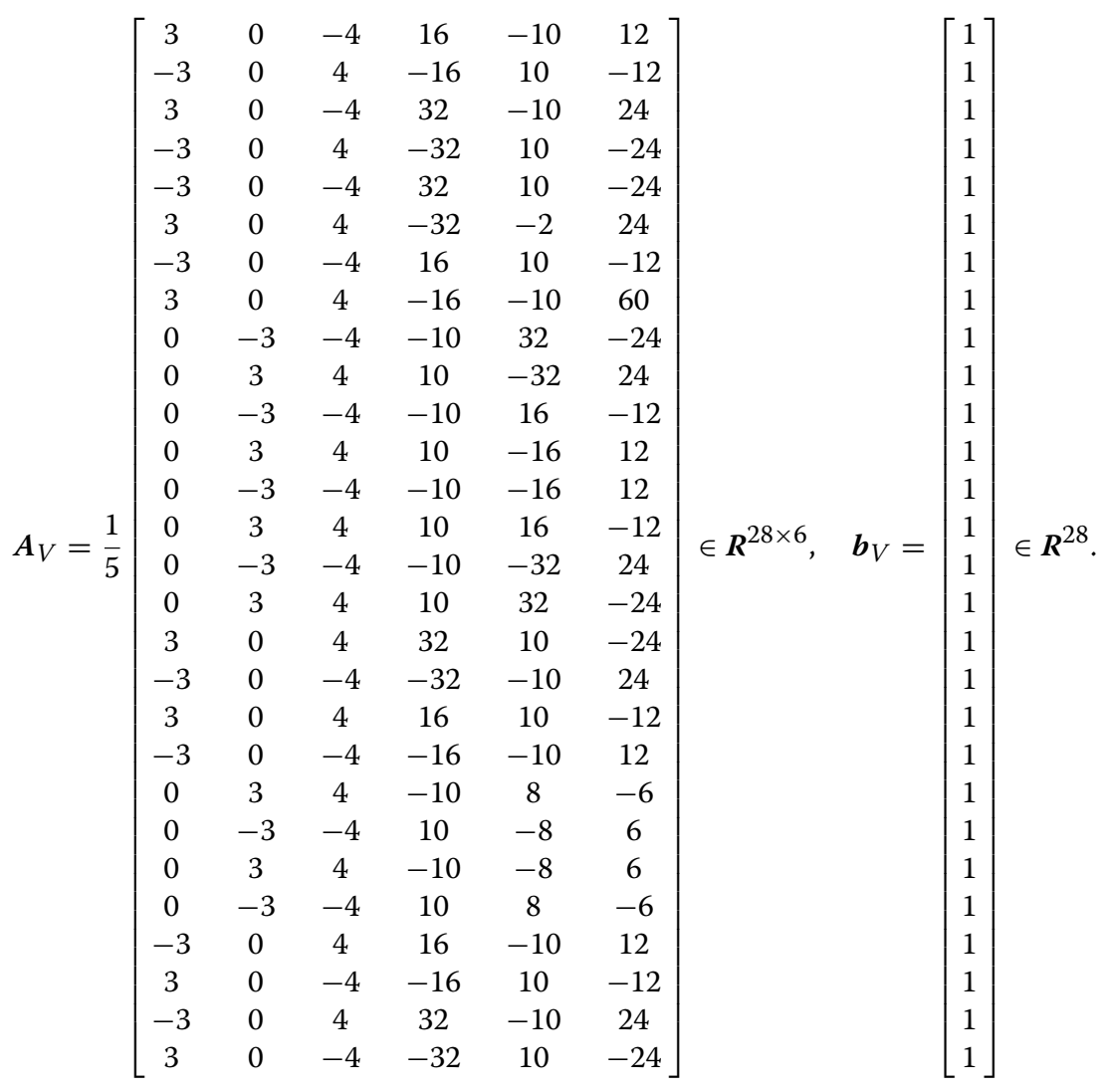




$$
\begin{aligned}
& { }^{T} \boldsymbol{p}_{B 11}=\left[\begin{array}{lll}
-8 & -9 & 8
\end{array}\right]^{\mathrm{T}}, \quad{ }^{T} \boldsymbol{p}_{B 12}=\left[\begin{array}{lll}
-4 & -9 & 4
\end{array}\right]^{\mathrm{T}}, \\
& { }^{\mathrm{T}} \boldsymbol{p}_{B 21}=\left[\begin{array}{lll}
4 & -9 & -4
\end{array}\right]^{\mathrm{T}}, \quad{ }^{\mathrm{T}} \boldsymbol{p}_{B 22}=\left[\begin{array}{lll}
8 & -9 & -8
\end{array}\right]^{\mathrm{T}}, \\
& { }^{\mathrm{T}} \boldsymbol{p}_{B 31}=\left[\begin{array}{lll}
9 & -8 & 8
\end{array}\right]^{\mathrm{T}}, \quad{ }^{\mathrm{T}} \boldsymbol{p}_{B 32}=\left[\begin{array}{llll}
9 & -4 & 4
\end{array}\right]^{\mathrm{T}}, \\
& { }^{T} \boldsymbol{p}_{B 41}=\left[\begin{array}{lll}
9 & 4 & -4
\end{array}\right]^{\mathrm{T}}, \quad{ }^{\mathrm{T}} \boldsymbol{p}_{B 42}=\left[\begin{array}{lll}
9 & 8 & -8
\end{array}\right]^{\mathrm{T}}, \\
& { }^{T} \boldsymbol{p}_{B 51}=\left[\begin{array}{lll}
8 & 9 & 8
\end{array}\right]^{\mathrm{T}}, \quad{ }^{\mathrm{T}} \boldsymbol{p}_{B 52}=\left[\begin{array}{lll}
4 & 9 & 4
\end{array}\right]^{\mathrm{T}}, \\
& { }^{T} \boldsymbol{p}_{B 61}=\left[\begin{array}{lll}
-4 & 9 & -4
\end{array}\right]^{\mathrm{T}}, \\
& { }^{T} \boldsymbol{p}_{B 71}=\left[\begin{array}{lll}
-9 & 8 & 8
\end{array}\right]^{\mathrm{T}}, \\
& { }^{T} \boldsymbol{p}_{B 62}=\left[\begin{array}{lll}
-8 & 9 & -8
\end{array}\right]^{\mathrm{T}}, \\
& { }^{T} \boldsymbol{p}_{B 72}=\left[\begin{array}{lll}
-9 & 4 & 4
\end{array}\right]^{\mathrm{T}}, \\
& { }^{T} \boldsymbol{p}_{B 81}=\left[\begin{array}{lll}
-9 & -4 & -4
\end{array}\right]^{\mathrm{T}}, \quad{ }^{T} \boldsymbol{p}_{B 82}=\left[\begin{array}{lll}
-9 & -8 & -8
\end{array}\right]^{\mathrm{T}} .
\end{aligned}
$$

Sets of positions ${ }^{B} p_{T}$ and orientations ${ }^{B} R_{T}$ of the top plate

${ }^{B} \boldsymbol{p}_{T}=\left[\begin{array}{lll}50 & 50 & 50\end{array}\right]^{\mathrm{T}}, \quad{ }^{B} \boldsymbol{R}_{T}=\left[\begin{array}{ccc}1 & 0 & 0 \\ 0 & 1 & 0 \\ 0 & 0 & 1\end{array}\right] \in \boldsymbol{R}^{3 \times 3}$.

\section{Positions of wire end points on the top plate}

From Eq. (73):

$$
\begin{array}{rlrl}
{ }^{B} \boldsymbol{p}_{B 11} & =\left[\begin{array}{lll}
42 & 41 & 58
\end{array}\right]^{\mathrm{T}}, & { }^{B} \boldsymbol{p}_{B 12} & =\left[\begin{array}{lll}
46 & 41 & 54
\end{array}\right]^{\mathrm{T}}, \\
{ }^{B} \boldsymbol{p}_{B 21} & =\left[\begin{array}{lll}
54 & 41 & 46
\end{array}\right]^{\mathrm{T}}, & { }^{B} \boldsymbol{p}_{B 22} & =\left[\begin{array}{lll}
58 & 41 & 42
\end{array}\right]^{\mathrm{T}}, \\
{ }^{B} \boldsymbol{p}_{B 31} & =\left[\begin{array}{lll}
59 & 42 & 58
\end{array}\right]^{\mathrm{T}}, & { }^{B} \boldsymbol{p}_{B 32} & =\left[\begin{array}{lll}
59 & 46 & 54
\end{array}\right]^{\mathrm{T}}, \\
{ }^{B} \boldsymbol{p}_{B 41} & =\left[\begin{array}{lll}
59 & 54 & 46
\end{array}\right]^{\mathrm{T}}, & { }^{B} \boldsymbol{p}_{B 42} & =\left[\begin{array}{llll}
59 & 58 & 42
\end{array}\right]^{\mathrm{T}}, \\
{ }^{B} \boldsymbol{p}_{B 51} & =\left[\begin{array}{lll}
58 & 59 & 58
\end{array}\right]^{\mathrm{T}}, & { }^{B} \boldsymbol{p}_{B 52}=\left[\begin{array}{llll}
54 & 59 & 54
\end{array}\right]^{\mathrm{T}}, \\
{ }^{B} \boldsymbol{p}_{B 61} & =\left[\begin{array}{lll}
46 & 59 & 46
\end{array}\right]^{\mathrm{T}}, & { }^{B} \boldsymbol{p}_{B 62}=\left[\begin{array}{llll}
42 & 59 & 42
\end{array}\right]^{\mathrm{T}}, \\
{ }^{B} \boldsymbol{p}_{B 71} & =\left[\begin{array}{lll}
41 & 58 & 58
\end{array}\right]^{\mathrm{T}}, & { }^{B} \boldsymbol{p}_{B 72} & =\left[\begin{array}{lll}
41 & 54 & 54
\end{array}\right]^{\mathrm{T}}, \\
{ }^{B} \boldsymbol{p}_{B 81} & =\left[\begin{array}{lll}
41 & 46 & 46
\end{array}\right]^{\mathrm{T}}, & { }^{B} \boldsymbol{p}_{B 82}=\left[\begin{array}{llll}
41 & 42 & 42
\end{array}\right]^{\mathrm{T}} .
\end{array}
$$

\section{Positions of wire end points on the frame}

The positions of the wire end points on the frame were ensured by appropriately arranging the VCMs on the frame:

$$
\begin{array}{rlrl}
{ }^{B} \boldsymbol{p}_{A 11} & =\left[\begin{array}{lll}
21.5 & 0 & 17
\end{array}\right]^{\mathrm{T}}, & { }^{B} \boldsymbol{p}_{A 12}=\left[\begin{array}{lll}
25.5 & 0 & 13
\end{array}\right]^{\mathrm{T}}, \\
{ }^{B} \boldsymbol{p}_{A 21} & =\left[\begin{array}{lll}
33.5 & 0 & 5
\end{array}\right]^{\mathrm{T}}, & { }^{B} \boldsymbol{p}_{A 22}=\left[\begin{array}{lll}
37.5 & 0 & 1
\end{array}\right]^{\mathrm{T}}, \\
{ }^{B} \boldsymbol{p}_{A 31} & =\left[\begin{array}{llll}
100 & 83 & 78.5
\end{array}\right]^{\mathrm{T}}, & { }^{B} \boldsymbol{p}_{A 32}=\left[\begin{array}{lll}
100 & 87 & 74.5
\end{array}\right]^{\mathrm{T}}, \\
{ }^{B} \boldsymbol{p}_{A 41} & =\left[\begin{array}{llll}
100 & 95 & 66.5
\end{array}\right]^{\mathrm{T}}, & { }^{B} \boldsymbol{p}_{A 42}=\left[\begin{array}{llll}
100 & 99 & 62.5
\end{array}\right]^{\mathrm{T}}, \\
{ }^{B} \boldsymbol{p}_{A 51} & =\left[\begin{array}{llll}
78.5 & 100 & 17
\end{array}\right]^{\mathrm{T}}, & { }^{B} \boldsymbol{p}_{A 52}=\left[\begin{array}{lll}
74.5 & 100 & 13
\end{array}\right]^{\mathrm{T}}, \\
{ }^{B} \boldsymbol{p}_{A 61}=\left[\begin{array}{llll}
66.5 & 100 & 5
\end{array}\right]^{\mathrm{T}}, & { }^{B} \boldsymbol{p}_{A 62}=\left[\begin{array}{llll}
62.5 & 100 & 1
\end{array}\right]^{\mathrm{T}}, \\
{ }^{B} \boldsymbol{p}_{A 71}=\left[\begin{array}{lll}
0 & 17 & 78.5
\end{array}\right]^{\mathrm{T}}, & { }^{B} \boldsymbol{p}_{A 72}=\left[\begin{array}{lll}
0 & 13 & 74.5
\end{array}\right]^{\mathrm{T}}, \\
{ }^{B} \boldsymbol{p}_{A 81}=\left[\begin{array}{lll}
0 & 5 & 66.5
\end{array}\right]^{\mathrm{T}}, & { }^{B} \boldsymbol{p}_{A 82}=\left[\begin{array}{lll}
0 & 1 & 62.5
\end{array}\right]^{\mathrm{T}} .
\end{array}
$$

\section{Calculating the wire vectors ${ }^{B} e_{i j}$}

The wire vectors ${ }^{B} \boldsymbol{e}_{i j}$ calculated by Eq. (74) are shown below:

$$
\begin{aligned}
&{ }^{B} \boldsymbol{e}_{11}={ }^{B} \boldsymbol{e}_{12}={ }^{B} \boldsymbol{e}_{21}={ }^{B} \boldsymbol{e}_{22}=\left[\begin{array}{lll}
-1 / 3 & -2 / 3 & -2 / 3
\end{array}\right]^{\mathrm{T}}, \\
&{ }^{B} \boldsymbol{e}_{31}={ }^{B} \boldsymbol{e}_{32}={ }^{B} \boldsymbol{e}_{41}={ }^{B} \boldsymbol{e}_{42}=\left[\begin{array}{lll}
2 / 3 & 2 / 3 & 1 / 3
\end{array}\right]^{\mathrm{T}}, \\
&{ }^{B} \boldsymbol{e}_{51}={ }^{B} \boldsymbol{e}_{52}={ }^{B} \boldsymbol{e}_{61}={ }^{B} \boldsymbol{e}_{62}=\left[\begin{array}{lll}
1 / 3 & 2 / 3 & -2 / 3
\end{array}\right]^{\mathrm{T}}, \\
&{ }^{B} \boldsymbol{e}_{71}={ }^{B} \boldsymbol{e}_{72}={ }^{B} \boldsymbol{e}_{81}={ }^{B} \boldsymbol{e}_{82}=\left[\begin{array}{lll}
-2 / 3 & -2 / 3 & 1 / 3
\end{array}\right]^{\mathrm{T}} .
\end{aligned}
$$

\section{KA (step 2)}

From Eq. (22), the matrix of total VCM contributions to the top plate's velocity is given by:

$$
\boldsymbol{A}_{L}=\operatorname{bdiag} .\left(\boldsymbol{A}_{L v 1}, \boldsymbol{A}_{L v 2}, \boldsymbol{A}_{L v 3}, \boldsymbol{A}_{L v 4}\right) \in \boldsymbol{R}^{32 \times 16} \text {. }
$$

The contents of $\boldsymbol{A}_{L v m}$ and $\boldsymbol{b}_{L v m}$ are shown in Eq. (16). The velocity matrices of the top plate $\boldsymbol{A}_{V}$ and $\boldsymbol{b}_{V}$, obtained by Eqs. (20) and (21) are given by: 


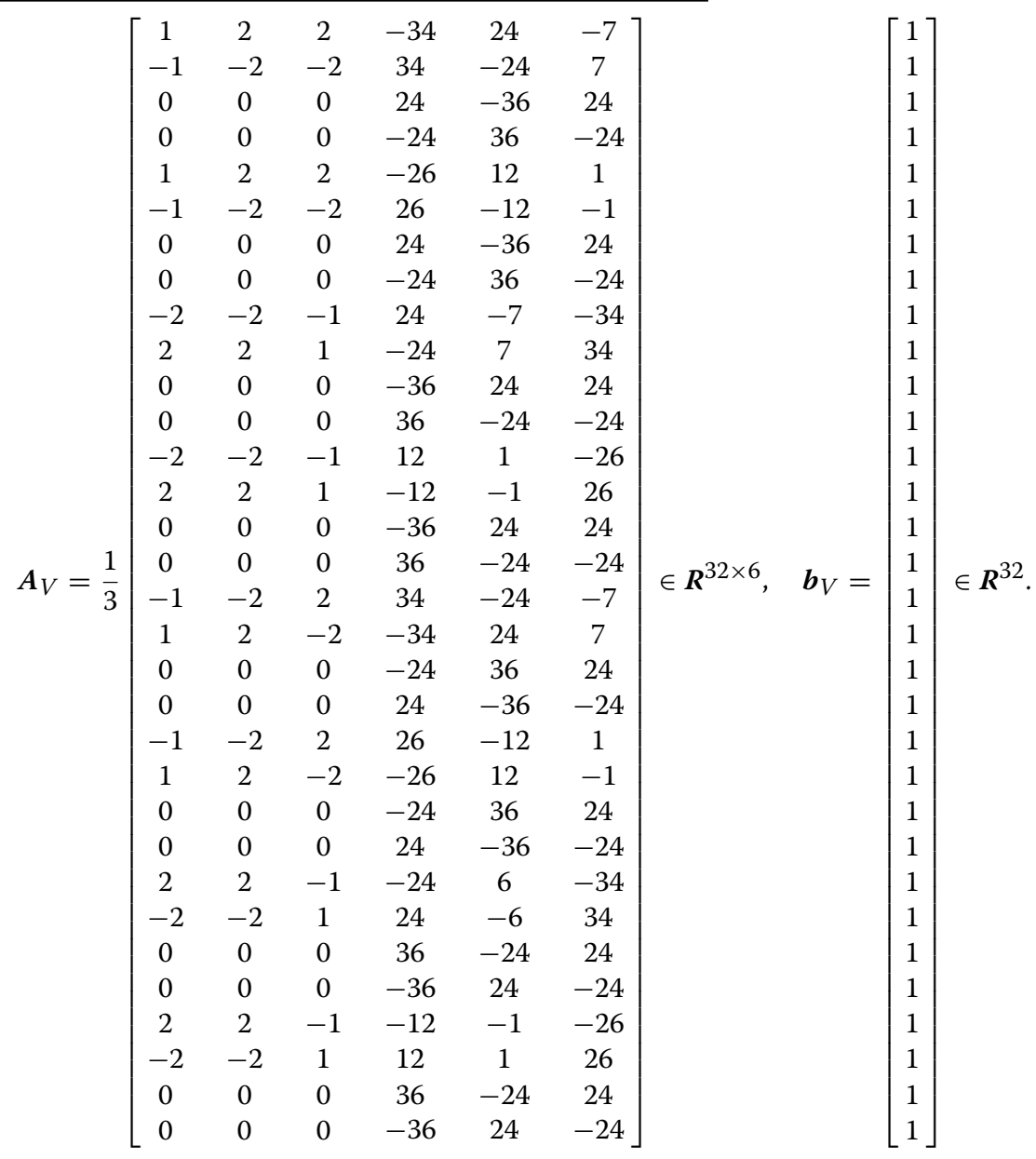

Solving the convex set in Eqs. (19) and (24) with the above $\boldsymbol{A}_{V}$ and $\boldsymbol{b}_{V}$, the vertex sets in matrix $\boldsymbol{A}$ of the 3D RDWM with four sets of VCM are calculated as:

$$
=\left\{\sum_{t=1}^{\beta} \lambda_{t} \mathrm{v}_{t} \mid \sum_{t=1}^{\beta} \lambda_{t} \leq 1, \lambda_{t} \geq 0, t \in[1, \beta]\right\},
$$

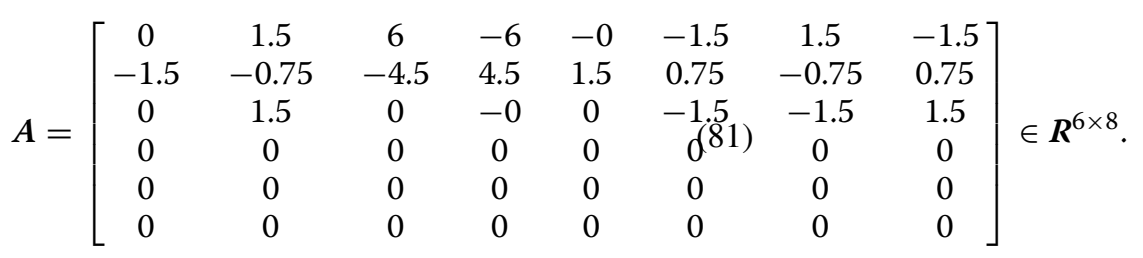

\section{Appendix 4: Conversion from face form to span} form

The face form is converted to span form as shown below:

$$
\boldsymbol{V}=\left\{\boldsymbol{v} \mid \boldsymbol{A}_{V} \boldsymbol{v} \leq \boldsymbol{b}_{V}\right\}
$$

Equations (82) and (83) express the face form with linear inequality sets and the span form with the vertex sets, respectively. The face and span forms are conceptualized in panels (A) and (B) respectively in Fig. 11, and the conversion is performed by the method proposed in [18]. 


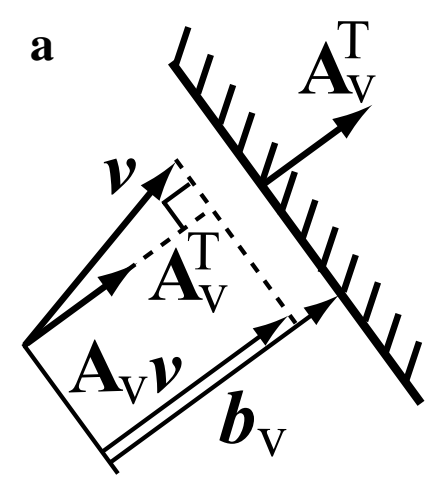

b

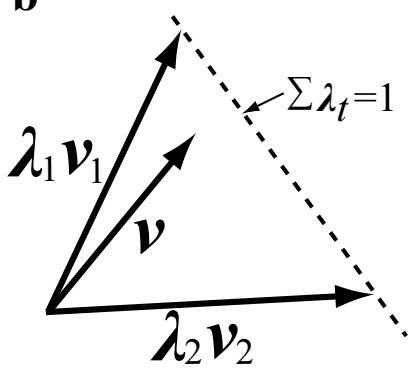

Fig. 11 Conversion from face form (a) and span form (b). a Shows the face form expression and $\mathbf{b}$ shows the span form expression

Received: 16 April 2016 Accepted: 30 August 2016 Published online: 23 September 2016

\section{References}

1. Kawamura S, Kino H, Won C (2000) High-speed manipulation by using parallel wire-driven robots. J Robot 18:13-21

2. Nagai K, Matsumoto M, Kimura K, Masuhara B (2003) Development of parallel manipulator 'NINJA' with ultra-high-acceleration. IEEE Int Conf Robot Autom 3:3678-3685

3. Vischer P, Clavel R (1998) Kinematic calibration of the parallel delta robot. Robotica 16:207-218
4. Pierrot F, Dauchez P, Fournier A (1991) HEXA: a fast six-DOF fully-parallel robot. Adv Robot 2:1158-1163

5. Nagai K, Nishibu Y, Dake K, Yamanaka A (2009) Design of a high speed parallel mechanism based on virtual force redundancy concept. IEEE Int Conf Ind Technol 1:1-6

6. Higashimori M, Kaneko M, Namiki A, Ishikawa M (2005) Design of 100G capturing robot based on dynamic preshaping. IEEE Int Conf Intell Robot Syst 24(9):743-753

7. Osumi H, Utsugi Y, Koshikawa M (2000) Development of a manipulator suspended by parallel wire structure. Proc 2000 IEEE/RSJ 1:498-503

8. Osumi H, Saitoh M (2000) Control of a redundant manipulator mounted on a base plate suspended by six wires. Proc 2006 IEEE/RSJ Int Conf Intell Robot Syst 1:73-78

9. Lampariello R, Heindl J, Koeppe R, Hirzinger G (2006) Reactionless control for two manipulators mounted on a cable-suspended platform. IEEE Int Conf Intell Robot Syst 1:91-97

10. Cong Pham B, Yeo SH, Yang G, Kurbanhusen MS, Chen I (2006) Forceclosure workspace analysis of cable-driven parallel mechanisms. Mech Mach Theory 41:53-69

11. Cong Pham B, Yeo SH, Yang G, Chen I (2009) Workspace analysis of fully restrained cable-driven manipulators. Robot Auton Syst 57:901-912

12. Van Nguyen D (1988) Constructing force closure grasps. Int J Robot Res 7(3):3-16

13. Nagai K, Le Nhat T, Hayashi Y, Ito K (2011) Proposal of redundant drive wire mechanism for producing motions with high acceleration and high precision. IEEE/SICE-international symposium on system intergration. IEEE, New York

14. Nagai K, Le Nhat T, Hayashi $Y$, Ito K (2012) Kinematical analysis of redundant drive wire mechanisms with velocity constraint. IEEE/international conference on mechatronics and automation. IEEE, New York, pp 1496-1501

15. Le Nhat T, Dobashi H, Nagai K (2016) Configuration of redundant drive wire mechanism using double actuator modules. ROBOMECH J, under-reviewing

16. Nagai $\mathrm{K}$, Yoshida H, Yoshimori D (2011) Kinematical analysis of parallel mechanism using multi-wire driven method. J Robot Soc Japan 29(9):65-72 (in Japanese)

17. Kino H, Yabe S, Kawamura S (2005) A force display system using a serial-link structure driven by a parallel-wire mechanism. Adv Robot 19(1):21-37

18. Hirukawa H, Matsui T, Takase K (1999) A fast algorithm for the analysis of the constraint for motion of polyhedra in contact and its application to departure motion planning. J Robot Soc Japan 9(7):841-848 (in Japanese)

19. Yoshikawa T (1999) Passive and active closures by constraining mechanisms. Trans ASME 121:418-424

\section{Submit your manuscript to a SpringerOpen ${ }^{\circ}$ journal and benefit from:}

- Convenient online submission

- Rigorous peer review

- Immediate publication on acceptance

- Open access: articles freely available online

- High visibility within the field

- Retaining the copyright to your article

Submit your next manuscript at springeropen.com 